\title{
Representation theory of the higher-order peak algebras
}

\author{
Jean-Christophe Novelli • Franco Saliola • \\ Jean-Yves Thibon
}

Received: 2 July 2009 / Accepted: 11 March 2010 / Published online: 7 April 2010

(C) Springer Science+Business Media, LLC 2010

\begin{abstract}
The representation theory (idempotents, quivers, Cartan invariants, and Loewy series) of the higher-order unital peak algebras is investigated. On the way, we obtain new interpretations and generating functions for the idempotents of descent algebras introduced in Saliola (J. Algebra 320:3866, 2008).
\end{abstract}

Keywords Noncommutative symmetric functions $\cdot$ Peak algebras · Finite dimensional algebras · Descent algebras

\section{Introduction}

A descent of a permutation $\sigma \in \mathfrak{S}_{n}$ is an index $i$ such that $\sigma(i)>\sigma(i+1)$. A descent is a peak if moreover $i>1$ and $\sigma(i)>\sigma(i-1)$. The sums of permutations with a given descent set span a subalgebra of the group algebra, the descent algebra $\Sigma_{n}$. The peak algebra $\stackrel{\circ}{\mathcal{P}}_{n}$ of $\mathfrak{S}_{n}$ is a subalgebra of its descent algebra, spanned by sums of permutations having the same peak set. This algebra has no unit.

The direct sum of the peak algebras is a Hopf subalgebra of the direct sum of all descent algebras, which can itself be identified with Sym, the Hopf algebra of noncommutative symmetric functions [11]. Actually, in [7], it was shown that most of the results on the peak algebras can be deduced from the case $q=-1$ of a $q$-identity

J.-C. Novelli $(\bowtie) \cdot$ F. Saliola · J.-Y. Thibon

Institut Gaspard Monge, Université Paris-Est Marne-la-Vallée, 5 Boulevard Descartes,

Champs-sur-Marne, 77454 Marne-la-Vallée cedex 2, France

e-mail: novelli@univ-mlv.fr

F. Saliola

e-mail: saliola@gmail.com

J.-Y. Thibon

e-mail: jyt@univ-mlv.fr 
of [14]. Specializing $q$ to other roots of unity, Krob and the third author introduced and studied higher-order peak algebras in [13]. Again, these are nonunital.

In [1], it has been shown that the peak algebra of $\mathfrak{S}_{n}$ can be naturally extended to a unital algebra which is obtained as a homomorphic image of the descent algebra of the hyperoctahedral group $B_{n}$. This construction has been extended in [3]. It is shown there that unital versions of the higher-order peak algebras can be obtained as homomorphic images of the Mantaci-Reutenauer algebras of type $B$.

Our purpose here is to investigate the representation theory of the unital higherorder peak algebras. The classical case has been worked out in [2]. In this reference, idempotents for the peak algebras were obtained from those of the descent algebras of type $B$ constructed in [6].

To deal with the general case, we need a different construction of idempotents. It turns out that the recursive algorithm introduced in [17] for idempotents of descent algebras can be adapted to higher-order peak algebras.

In order to achieve this, we need a better understanding of the idempotents generated by the algorithm of [17]. Interpreting them as noncommutative symmetric functions, we find that in type $A$, these idempotents are associated with a known family of Lie idempotents, the so-called Zassenhaus idempotents, by the construction of [14]. We then show that similar Lie idempotents can be defined in type $B$ as well, which yields a simple generating function in terms of noncommutative symmetric functions of type $B$.

This being understood, we obtain complete families of orthogonal idempotents for the higher-order peak algebras, which can be described either by recurrence relations as in [17] or by generating series of noncommutative symmetric functions.

Finally, we make use of these idempotents to study the quivers, Cartan invariants, and the Loewy series of the unital higher-order peak algebras.

\section{Notations and background}

\subsection{Noncommutative symmetric functions}

We will assume familiarity with the standard notations of the theory of noncommutative symmetric functions [11] and with the main results of [3, 13]. We recall here only a few essential definitions.

The Hopf algebra of noncommutative symmetric functions is denoted by Sym, or by $\operatorname{Sym}(A)$ if we consider the realization in terms of an auxiliary alphabet $A$. Linear bases of $\mathbf{S y m}_{n}$ are labeled by compositions $I=\left(i_{1}, \ldots, i_{r}\right)$ of $n$ (we write $I \vDash n$ ). The noncommutative complete and elementary functions are denoted by $S_{n}$ and $\Lambda_{n}$, and $S^{I}=S_{i_{1}} \cdots S_{i_{r}}$. The ribbon basis is denoted by $R_{I}$. The descent set of $I$ is $\operatorname{Des}(I)=$ $\left\{i_{1}, i_{1}+i_{2}, \ldots, i_{1}+\cdots+i_{r-1}\right\}$. The descent composition of a permutation $\sigma \in \mathfrak{S}_{n}$ is the composition $I$ of $n$ whose descent set is the descent set $D(\sigma)$ of $\sigma$.

\subsection{The Mantaci-Reutenauer algebra of type $B$}

We denote by MR the free product $\mathbf{S y m} \star \mathbf{S y m}$ of two copies of the Hopf algebra of noncommutative symmetric functions [15]. That is, $\mathbf{M R}$ is the free associative algebra on two sequences $\left(S_{n}\right)$ and $\left(S_{\bar{n}}\right)(n \geq 1)$. We regard the two copies of Sym 
as noncommutative symmetric functions on two auxiliary alphabets: $S_{n}=S_{n}(A)$ and $S_{\bar{n}}=S_{n}(\bar{A})$. We denote by $F \mapsto \bar{F}$ the involutive antiautomorphism which exchanges $S_{n}$ and $S_{\bar{n}}$. The bialgebra structure is defined by the requirement that the series

$$
\sigma_{1}=\sum_{n \geq 0} S_{n} \quad \text { and } \quad \bar{\sigma}_{1}=\sum_{n \geq 0} S_{\bar{n}}
$$

are grouplike. The internal product of $\mathbf{M R}$ can be computed from the splitting formula

$$
\left(f_{1} \cdots f_{r}\right) * g=\mu_{r} \cdot\left(f_{1} \otimes \cdots \otimes f_{r}\right) *_{r} \Delta^{r} g,
$$

where $\mu_{r}$ is $r$-fold multiplication, and $\Delta^{r}$ the iterated coproduct with values in the $r$ th tensor power and the conditions: $\sigma_{1}$ is neutral, $\bar{\sigma}_{1}$ is central, and $\bar{\sigma}_{1} * \bar{\sigma}_{1}=\sigma_{1}$.

\subsection{Noncommutative symmetric functions of type $B$}

Noncommutative symmetric functions of type $B$ were introduced in [9] as the right Sym-module BSym freely generated by another sequence $\left(\tilde{S}_{n}\right)\left(n \geq 0, \tilde{S}_{0}=1\right)$ of homogeneous elements, with $\tilde{\sigma}_{1}$ grouplike. This is a coalgebra, but not an algebra. It is endowed with an internal product for which each homogeneous component $\mathbf{B S y m}_{n}$ is antiisomorphic to the descent algebra of $B_{n}$.

It should be noted that with this definition, the restriction of the internal product of BSym to Sym is not the internal product of Sym. To remedy this inconvenience, we use a different realization of BSym. We embed BSym as a subcoalgebra and sub-Sym-module of MR as follows. Define, for $F \in \operatorname{Sym}(A)$,

$$
F^{\sharp}=F(A \mid \bar{A})=\left.F(A-q \bar{A})\right|_{q=-1}
$$

called the supersymmetric version, or superization, of $F$ [16]. It is also equal to

$$
F^{\sharp}=F * \sigma_{1}^{\sharp} \text {. }
$$

Indeed, $\sigma_{1}^{\sharp}$ is grouplike, and for $F=S^{I}$, the splitting formula gives

$$
\left(S_{i_{1}} \cdots S_{i_{r}}\right) * \sigma_{1}^{\sharp}=\mu_{r}\left[\left(S_{i_{1}} \otimes \cdots \otimes S_{i_{r}}\right) *\left(\sigma_{1}^{\sharp} \otimes \cdots \otimes \sigma_{1}^{\sharp}\right)\right]=S^{I \sharp} .
$$

We have

$$
\sigma_{1}^{\sharp}=\bar{\lambda}_{1} \sigma_{1}=\sum \Lambda_{i} S_{j} .
$$

The element $\bar{\sigma}_{1}$ is central for the internal product, and

$$
\bar{\sigma}_{1} * F(A, \bar{A})=F(\bar{A}, A)=F * \bar{\sigma}_{1} .
$$

The basis element $\tilde{S}^{I}$ of $\mathbf{B S y m}$, where $I=\left(i_{0}, i_{1}, \ldots, i_{r}\right)$ is a $B$-composition (that is, $i_{0}$ may be 0$)$, can be embedded as

$$
\tilde{S}^{I}=S_{i_{0}}(A) S^{i_{1} i_{2} \cdots i_{r}}(A \mid \bar{A}) .
$$

We will identify BSym with its image under this embedding. 


\subsection{Other notation}

For a partition $\lambda$, we denote by $m_{i}(\lambda)$ the multiplicity of $i$ in $\lambda$ and set $m_{\lambda}:=$ $\prod_{i \geq 1} m_{i}(\lambda)$ !.

The reverse refinement order on compositions is denoted by $\preceq$. The nonincreasing rearrangement of a composition is denoted by $I \downarrow$. The refinement order on partitions is denoted by $\prec_{p}: \lambda \prec_{p} \mu$ if $\lambda$ is finer than $\mu$, that is, each part of $\mu$ is a sum of parts of $\lambda$.

\section{Descent algebras of type $A$}

\subsection{Principal idempotents}

In [17], a recursive construction of complete sets of orthogonal idempotents of descent algebras has been described. In [14], one finds a general method for constructing such families from an arbitrary sequence of Lie idempotents, as well as many remarkable families of Lie idempotents. It is therefore natural to investigate whether the resulting idempotents can be derived from a (possibly known) sequence of Lie idempotents. We shall show that it is indeed the case.

Let $P_{n}$ be the sequence of partitions of $n$ ordered in the following way: first, sort them by decreasing length, then, for each length, order them by reverse lexicographic order. We denote this order by $\leq$. For example,

$$
P_{5}=[11111,2111,311,221,41,32,5] .
$$

Now, start with

$$
e_{1^{n}}:=\frac{1}{n !} S_{1}^{n}
$$

and define by induction

$$
e_{\lambda}:=\frac{1}{m_{\lambda}} S^{\lambda} *\left(S_{n}-\sum_{\mu<\lambda} e_{\mu}\right)
$$

Theorem 3.1 [17] The family $\left(e_{\lambda}\right)_{\lambda \vdash n}$ forms a complete system of orthogonal idempotents for $\mathbf{S y m}_{n}$.

Following [14], define the (left) Zassenhaus idempotents $\zeta_{n}$ by the generating series

$$
\sigma_{1}=: \overleftarrow{\prod_{k \geq 1}} e^{\zeta_{k}}=\cdots e^{\zeta_{3}} e^{\zeta_{2}} e^{\zeta_{1}}
$$

For example,

$$
S_{1}=\zeta_{1}, \quad S_{2}=\zeta_{2}+\frac{1}{2} \zeta_{1}^{2}, \quad S_{3}=\zeta_{3}+\zeta_{2} \zeta_{1}+\frac{1}{6} \zeta_{1}^{3},
$$




$$
\begin{aligned}
S_{4}= & \zeta_{4}+\zeta_{3} \zeta_{1}+\frac{1}{2} \zeta_{2}^{2}+\frac{1}{2} \zeta_{2} \zeta_{1}^{2}+\frac{1}{24} \zeta_{1}^{4}, \\
S_{5}= & \zeta_{5}+\zeta_{4} \zeta_{1}+\zeta_{3} \zeta_{2}+\frac{1}{2} \zeta_{3} \zeta_{1}^{2}+\frac{1}{2} \zeta_{2}^{2} \zeta_{1}+\frac{1}{6} \zeta_{2} \zeta_{1}^{3}+\frac{1}{120} \zeta_{1}^{5} \\
S_{6}= & \zeta_{6}+\zeta_{5} \zeta_{1}+\zeta_{4} \zeta_{2}+\frac{1}{2} \zeta_{4} \zeta_{1}^{2}+\frac{1}{2} \zeta_{3}^{2}+\zeta_{3} \zeta_{2} \zeta_{1} \\
& +\frac{1}{6} \zeta_{3} \zeta_{1}^{3}+\frac{1}{6} \zeta_{2}^{3}+\frac{1}{4} \zeta_{2}^{2} \zeta_{1}^{2}+\frac{1}{24} \zeta_{2} \zeta_{1}^{4}+\frac{1}{720} \zeta_{1}^{6}
\end{aligned}
$$

so that

$$
\begin{aligned}
\zeta_{1}= & S_{1}, \quad \zeta_{2}=S_{2}-\frac{1}{2} S^{11}, \quad \zeta_{3}=S_{3}-S^{21}+\frac{1}{3} S^{111}, \\
\zeta_{4}= & S_{4}-S^{31}-\frac{1}{2} S^{22}+\frac{3}{4} S^{211}+\frac{1}{4} S^{112}-\frac{1}{4} S^{1111} \\
\zeta_{5}= & S_{5}-S^{41}-S^{32}+S^{311}+S^{212}-\frac{2}{3} S^{2111}-\frac{1}{3} S^{1112}+\frac{1}{5} S^{11111}, \\
\zeta_{6}= & S_{6}-S^{51}-S^{42}+S^{411}-\frac{1}{2} S^{33}+\frac{1}{2} S^{321}+S^{312}-\frac{5}{6} S^{3111} \\
& +\frac{1}{3} S^{222}-\frac{1}{6} S^{2211}+\frac{1}{2} S^{213}-\frac{1}{2} S^{2121}-\frac{2}{3} S^{2112}+\frac{13}{24} S^{21111} \\
& -\frac{1}{6} S^{1122}+\frac{1}{12} S^{11211}-\frac{1}{6} S^{1113}+\frac{1}{6} S^{11121}+\frac{5}{24} S^{11112}-\frac{1}{6} S^{111111} .
\end{aligned}
$$

Note that, in particular,

$$
S_{n}=\sum_{\lambda \vdash n} \frac{1}{m_{\lambda}} \zeta_{\lambda_{1}} \zeta_{\lambda_{2}} \cdots \zeta_{\lambda_{r}}
$$

For a composition $I=\left(i_{1}, \ldots, i_{r}\right)$, define as usual $\zeta^{I}:=\zeta_{i_{1}} \cdots \zeta_{i_{r}}$. Since $\zeta_{n} \equiv S_{n}$ modulo smaller terms in the refinement order on compositions, $\zeta^{I} \equiv S^{I}$ modulo smaller terms. So the $\zeta^{I}$ family is unitriangular on the basis $S^{J}$, so it is a basis of Sym.

In the sequel, we shall need a condition for a product $S^{I} * \zeta^{J}$ to be zero.

Lemma 3.2 Let I and $J$ be two compositions of $n$. Then,

$$
S^{I} * \zeta^{J}= \begin{cases}0 & \text { if } J \downarrow \nprec_{p} I \downarrow, \\ m_{I \downarrow} \zeta^{I} & \text { if } J \downarrow=I \downarrow, \\ \sum_{K \downarrow=J \downarrow} c_{I J}^{K} \zeta^{K} & \text { otherwise }\end{cases}
$$

where $c_{I J}^{K}$ is the number of ways of unshuffling $J$ into $p=\ell(I)$ subwords such that $J^{(l)}$ has sum $i_{l}$ and whose concatenation $J^{(1)} J^{(2)} \ldots J^{(p)}$ is $K$. 
Proof Since the Zassenhaus idempotents $\zeta_{m}$ are primitive, we have, thanks to the splitting formula (2),

$$
S^{I} * \zeta^{J}=\sum_{J^{(1)}, \ldots, J(p)}\left(S_{i_{1}} * \zeta^{J^{(1)}}\right) \cdots\left(S_{i_{p}} * \zeta^{J^{(p)}}\right)
$$

where the sum ranges over all possible ways of decomposing $J$ into $p$ (possibly empty) subwords.

Since $S_{i_{j}} * \zeta^{J^{(j)}}=0$ if $J^{(j)}$ is not a composition of $i_{j}$, it follows that $S^{I} * \zeta^{J}=0$ if $J \downarrow \nprec_{p} I \downarrow$. Moreover, if $J \downarrow=I \downarrow$, then

$$
S^{I} * \zeta^{J}=\sum_{\sigma \in \mathfrak{S}_{p}}\left(S_{i_{1}} * \zeta_{J_{\sigma(1)}}\right) \cdots\left(S_{i_{p}} * \zeta_{J_{\sigma(p)}}\right)=m_{I} \zeta^{I}
$$

If $J \downarrow \prec_{p} I \downarrow$ and $J \downarrow \neq I \downarrow$, then a term in the r.h.s. of (23) is nonzero iff all $J^{(\ell)}$ are compositions of $i_{\ell}$. In that case, we have

$$
S^{I} * \zeta^{J}=\sum_{J^{(1)}, \ldots, J^{(p)}} \zeta^{J^{(1)}} \cdots \zeta^{J^{(p)}}=\sum_{J^{(1)}, \ldots, J^{(p)}} \zeta^{J^{(1)} J^{(2)} \ldots J^{(p)}}
$$

whence the last case.

Theorem 3.3 For all partitions $\lambda=\left(\lambda_{1}, \lambda_{2}, \ldots, \lambda_{k}\right)$,

$$
e_{\lambda}=\frac{1}{m_{\lambda}} \zeta_{\lambda_{1}} \cdots \zeta_{\lambda_{k}}
$$

Proof Let $e_{\lambda}^{\prime}$ be the right-hand side of (26). We will show that these elements satisfy the same induction as the $e_{\lambda}(11)$.

From Lemma 3.2 we have

$$
S^{\lambda} * e_{\lambda}^{\prime}=m_{\lambda} e_{\lambda}^{\prime}
$$

Now, using (21), we get

$$
m_{\lambda} e_{\lambda}^{\prime}=S^{\lambda} *\left(S_{n}-\sum_{\mu \neq \lambda} e_{\mu}^{\prime}\right)=S^{\lambda} *\left(S_{n}-\sum_{\mu<\lambda} e_{\mu}^{\prime}\right)
$$

where the last equality follows again from Lemma 3.2. Hence, $e_{\lambda}=e_{\lambda}^{\prime}$.

Note that, thanks to Lemma 3.2, the induction formula for $e_{\lambda}$ simplifies to

$$
e_{\lambda}=\frac{1}{m_{\lambda}} S^{\lambda} *\left(S_{n}-\sum_{\mu \prec_{p} \lambda} e_{\mu}\right)
$$




\subsection{A basis of idempotents}

As with any sequence of Lie idempotents, we can construct an idempotent basis of $\mathbf{S y m}_{n}$ from the $\zeta_{n}$. Here, the principal idempotents $e_{\lambda}$ are members of the basis, which leads to a simpler derivation of the representation theory.

We start with a basic lemma, easily derived from the splitting formula (compare [14, Lemma 3.10]). Recall that the radical of $\left(\mathbf{S y m}_{n}, *\right)$ is $\mathcal{R}_{n}=\mathcal{R} \cap \mathbf{S y m}_{n}$, where $\mathcal{R}$ is the kernel of the commutative image $\mathbf{S y m} \rightarrow$ Sym.

Lemma 3.4 Denote by $\mathfrak{S}(J)$ the set of distinct rearrangements of a composition $J$. Let $I=\left(i_{1}, \ldots, i_{r}\right)$ and $J=\left(j_{1}, \ldots, j_{s}\right)$ be two compositions of $n$.Then,

(i) If $\ell(J)<\ell(I)$, then $\zeta^{I} * \zeta^{J}=0$.

(ii) If $\ell(J)>\ell(I)$, then $\zeta^{I} * \zeta^{J} \in \operatorname{Vect}\left\langle\zeta^{K}: K \in \mathfrak{S}(J)\right\rangle \cap \mathcal{R}$. More precisely,

$$
\zeta^{I} * \zeta^{J}=\sum_{\substack{J_{1}, \ldots, J_{r} \\\left|J_{k}\right|=i_{k}}}\left\langle J, J_{1} \uplus \cdots \uplus J_{r}\right\rangle \Gamma_{J_{1}} \cdots \Gamma_{J_{r}},
$$

where for a composition $K$ of $k, \Gamma_{K}:=\zeta_{k} * \zeta^{K}$.

(iii) If $\ell(J)=\ell(I)$, then $\zeta^{I} * \zeta^{J} \neq 0$ only for $J \in \mathfrak{S}(I)$, in which case $\zeta^{I} * \zeta^{J}=$ $m_{I} \zeta^{I}$

Note that the $\Gamma_{K}$ are in the primitive Lie algebra. This follows from the $*$-multiplicativity of the coproduct: $\Delta(f * g)=\Delta(f) * \Delta(g)$, see [11, Proposition 5.5].

Corollary 3.5 The elements

$$
e_{I}=\frac{1}{m_{I}} \zeta^{I}, \quad I \vDash n,
$$

are all idempotents and form a basis of $\mathbf{S y m}_{n}$. This basis contains in particular the principal idempotents $e_{\lambda}$.

\subsection{Cartan invariants}

By (iii) of Lemma 3.4, the indecomposable projective module $P_{\lambda}=\mathbf{S y m}_{n} * e_{\lambda}$ contains the $e_{I}$ for $I \in \mathfrak{S}(\lambda)$. For $I \notin \mathfrak{S}(\lambda)$, (i) and (ii) imply that $e_{I} * e_{\lambda}$ is in $\operatorname{Vect}\left\langle\zeta^{K}: K \in \mathfrak{S}(\lambda)\right\rangle$. Hence, this space coincides with $P_{\lambda}$. So, we get immediately the explicit decomposition

$$
\mathbf{S y m}_{n}=\bigoplus_{\lambda \vdash n} P_{\lambda}, \quad P_{\lambda}=\bigoplus_{I \in \mathfrak{S}(\lambda)} \mathbb{C} e_{I} .
$$

The Cartan invariants

$$
c_{\lambda \mu}=\operatorname{dim}\left(e_{\mu} * \mathbf{S y m}_{n} * e_{\lambda}\right)
$$


are also easily obtained. The above space is spanned by the

$$
e_{\mu} * e_{I} * e_{\lambda}=e_{\mu} * e_{I}, \quad I \in \mathfrak{S}(\lambda) .
$$

By (ii) of Lemma 3.4, this is the dimension of the space $\left[S^{\mu}(L)\right]_{\lambda}$ spanned by all symmetrized products of Lie polynomials of degrees $\mu_{1}, \mu_{2}, \ldots$ formed from $\zeta_{i_{1}}, \zeta_{i_{2}}, \ldots$, hence giving back the classical result of Garsia-Reutenauer [10].

\subsection{Quiver and $q$-Cartan invariants (Loewy series)}

Still relying upon point (ii) of Lemma 3.4, we see that $c_{\lambda \mu}=0$ if $\lambda$ is not finer than (or equal to) $\mu$. Further, if $\mu$ is obtained from $\lambda$ by adding up two parts $\lambda_{i}, \lambda_{j}$, then $e_{\mu} * e_{I}=0$ if $\lambda_{i}=\lambda_{j}$ and is a nonzero element of the radical otherwise.

In [8], it is shown that the powers of the radical for the internal product coincide with the lower central series of Sym for the external product:

$$
\mathcal{R}^{* j}=\gamma^{j}(\mathbf{S y m}),
$$

where $\gamma^{j}(\mathbf{S y m})$ is the ideal generated by the commutators [Sym, $\left.\gamma^{j-1}(\mathbf{S y m})\right]$. Hence, for $\lambda$ finer than $\mu, e_{\mu} * e_{I}$ is nonzero modulo $\mathcal{R}^{* 2}$ iff $\mu$ is obtained from $\lambda$ by summing two distinct parts. More generally, $e_{\mu} * e_{I}$ is in $\mathcal{R}^{* k}$ and nonzero modulo $\mathcal{R}^{* k+1}$ iff $\ell(\lambda)-\ell(\mu)=k$.

Summarizing, we have the following:

Theorem $3.6[8,17]$

(i) In the quiver of $\mathbf{S y m}_{n}$, there is an arrow $\lambda \rightarrow \mu$ iff $\mu$ is obtained from $\lambda$ by adding two distinct parts.

(ii) The q-Cartan invariants are given by

$$
c_{\lambda \mu}(q)=q^{\ell(\lambda)-\ell(\mu)}
$$

if $\lambda$ is finer than (or equal to) $\mu$ and $c_{\lambda \mu}(q)=0$ otherwise.

\section{Descent algebras of type $B$}

\subsection{Reinterpretation of some results of Chow}

We begin by showing that in our realization of BSym, Chow's map $\Theta$ (see [9, Sect. 3.4]) corresponds to the left internal product by the reproducing kernel $\sigma_{1}^{\sharp}$ of the superization map. Chow's condition $\Theta\left(\tilde{S}_{n}\right)=S_{n}$ translates into the obvious equality $\sigma_{1}^{\sharp} * \sigma_{1}=\sigma_{1}^{\sharp}$. The second condition $\Theta\left(S_{n}(A)\right)=S_{n}(2 A)$ amounts to

$$
\sigma_{1}^{\sharp} * \sigma_{1}^{\sharp}=\left(\sigma_{1}^{\sharp}\right)^{2},
$$

which is an easy consequence of the splitting formula

$$
\sigma_{1}^{\sharp} * \sigma_{1}^{\sharp}=\left(\bar{\lambda}_{1} \sigma_{1}\right) * \sigma_{1}^{\sharp}=\left(\bar{\lambda}_{1} * \sigma_{1}^{\sharp}\right) \sigma_{1}^{\sharp}=\left(\sigma_{1}^{\sharp}\right)^{2}
$$


since

$$
\bar{\lambda}_{1} * \sigma_{1}^{\sharp}=\left(\bar{\lambda}_{1} * \sigma_{1}\right)\left(\bar{\lambda}_{1} * \bar{\lambda}_{1}\right)=\sigma_{1}^{\sharp} .
$$

Here we used the fact that left $*$-multiplication by $\bar{\lambda}_{1}$ is an antiautomorphism. Denoting by $\mu^{\prime}$ as in [9] the twisted product

$$
\mu^{\prime}(A \otimes B \otimes C)=\left(\bar{\lambda}_{1} * B\right) A C,
$$

we have the following:

Lemma 4.1 For $F, G \in \mathbf{B S y m}$,

$$
\sigma_{1}^{\sharp} *(F G)=\mu^{\prime}\left[\left(\sigma_{1}^{\sharp} * F\right) \otimes \Delta(G)\right] .
$$

Proof

$$
\begin{aligned}
\sigma_{1}^{\sharp} *(F G) & =\mu\left[\left(\bar{\lambda}_{1} \otimes \sigma_{1}\right) *(\Delta F \Delta G)\right]=\sum_{(F),(G)} \mu\left[\left(\bar{\lambda}_{1} * F_{1} G_{1}\right) \otimes F_{2} G_{2}\right] \\
& =\sum\left(\bar{\lambda}_{1} * G_{1}\right)\left(\left(\bar{\lambda}_{1} * F_{1}\right) F_{2}\right) G_{2}=\mu^{\prime}\left[\left(\sigma_{1}^{\sharp} * F\right) \otimes \Delta(G)\right] .
\end{aligned}
$$

This is Chow's third condition, which completes the characterization of $\Theta$.

\subsection{Idempotents in BSym}

Define the elements $\zeta_{n} \in \mathbf{B S y m}$ by the generating series

$$
\sigma_{1}^{\sharp}=:\left(e^{\zeta_{1}} e^{\zeta_{2}} e^{\zeta_{3}} \cdots\right)\left(\cdots e^{\zeta_{3}} e^{\zeta_{2}} e^{\zeta_{1}}\right) .
$$

For example, collecting the terms of weights 1, 2, and 3, respectively, we have

$$
S_{1}^{\sharp}=2 \zeta_{1}, \quad S_{2}^{\sharp}=2 \zeta_{2}+2 \zeta_{1}^{2}, \quad S_{3}^{\sharp}=2 \zeta_{3}+2 \zeta_{2} \zeta_{1}+2 \zeta_{1} \zeta_{2}+\frac{4}{3} \zeta_{1}^{3},
$$

so that

$$
\zeta_{1}=\frac{1}{2} S_{1}^{\sharp}, \quad \zeta_{2}=\frac{1}{2} S_{2}^{\sharp}-\frac{1}{4} S^{11 \sharp}, \quad \zeta_{3}=\frac{1}{2} S_{3}^{\sharp}-\frac{1}{4} S^{21 \sharp}-\frac{1}{4} S^{12 \sharp}+\frac{1}{6} S^{111 \sharp} .
$$

Note that the elements $\zeta_{n}$ are well defined and that they are primitive. We shall use the notation

$$
\left(e^{\zeta_{1}} e^{\zeta_{2}} \cdots\right)=: \mathcal{E}^{\uparrow}(\zeta), \quad\left(\cdots e^{\zeta_{2}} e^{\zeta_{1}}\right)=: \mathcal{E}^{\downarrow}(\zeta)
$$

Next, define the elements $\tilde{\zeta}_{n} \in \mathbf{B S y m}$ by the generating series

$$
\sigma_{1}=:\left(\sum_{n \geq 0} \tilde{\zeta}_{n}\right)\left(\cdots e^{\zeta_{2}} e^{\zeta_{1}}\right)=: \tilde{\zeta} \mathcal{E}^{\downarrow}(\zeta)
$$


For example,

$$
\begin{aligned}
& S_{1}=\zeta_{1}+\tilde{\zeta}_{1}, \quad S_{2}=\zeta_{2}+\frac{1}{2} \zeta_{1}^{2}+\tilde{\zeta}_{1} \zeta_{1}+\tilde{\zeta}_{2}, \\
& S_{3}=\frac{1}{6} \zeta_{1}^{3}+\zeta_{2} \zeta_{1}+\zeta_{3}+\tilde{\zeta}_{1} \zeta_{2}+\frac{1}{2} \tilde{\zeta}_{1} \zeta_{1}^{2}+\tilde{\zeta}_{2} \zeta_{1}+\tilde{\zeta}_{3},
\end{aligned}
$$

so that

$$
\begin{aligned}
& \tilde{\zeta}_{1}=S_{1}-\frac{1}{2} S_{1}^{\sharp}, \quad \tilde{\zeta}_{2}=S_{2}-\frac{1}{2} S_{2}^{\sharp}-\frac{1}{2} S_{1} S_{1}^{\sharp}+\frac{3}{8} S^{11 \sharp}, \\
& \tilde{\zeta}_{3}=S_{3}-\frac{1}{2} S_{2} S_{1}^{\sharp}-\frac{1}{2} S_{1} S_{2}^{\sharp}+\frac{3}{8} S_{1} S^{11 \sharp}-\frac{1}{2} S_{3}^{\sharp}+\frac{1}{4} S^{21 \sharp}+\frac{1}{2} S^{12 \sharp}-\frac{5}{16} S^{111 \sharp} .
\end{aligned}
$$

Since $\sigma_{1}$ is grouplike and since $e^{\zeta_{n}}$ is grouplike for all $n \geq 1$, the series $\tilde{\zeta}$ is also grouplike.

The next two lemmas describe some properties of the elements $\tilde{\zeta}_{n}$ and $\zeta_{n}$.

Lemma 4.2 The ordered exponentials are exchanged as follows:

$$
\bar{\lambda}_{1} * \mathcal{E}^{\downarrow}(\zeta)=\mathcal{E}^{\uparrow}(\zeta)
$$

In particular, $\bar{\lambda}_{1} * \zeta_{i}=\zeta_{i}$ for all $i \geq 0$.

Proof $\bar{\lambda}_{1} * \cdot$ is an antiautomorphism, so the left-hand side is

$$
\left(\bar{\lambda}_{1} * e^{\zeta_{1}}\right)\left(\bar{\lambda}_{1} * e^{\zeta_{2}}\right) \cdots
$$

Taking into account (39) and recalling that (43) characterizes the $\zeta_{i}$, we see that if we set

$$
\zeta_{i}^{\prime}:=\bar{\lambda}_{1} * \zeta_{i}
$$

then

$$
\sigma_{1}^{\sharp}=\left(e^{\zeta_{1}^{\prime}} e^{\zeta_{2}^{\prime}} \ldots\right)\left(\cdots e^{\zeta_{2}^{\prime}} e^{\zeta_{1}^{\prime}}\right),
$$

so that $\zeta_{i}^{\prime}=\zeta_{i}$.

Lemma 4.3 For all $n \geq 1$,

$$
\sigma_{1}^{\sharp} * \tilde{\zeta}_{n}=0 .
$$

Proof By definition,

$$
\begin{aligned}
\mathcal{E}^{\uparrow}(\zeta) \mathcal{E}^{\downarrow}(\zeta) & =\sigma_{1}^{\sharp}=\sigma_{1}^{\sharp} * \sigma_{1}=\sigma_{1}^{\sharp} *\left[\tilde{\zeta} \mathcal{E}^{\downarrow}(\zeta)\right]=\left(\bar{\lambda}_{1} \sigma_{1}\right) *\left(\tilde{\zeta} \mathcal{E}^{\downarrow}(\zeta)\right) \\
& =\mu\left[\left(\bar{\lambda}_{1} \otimes \sigma_{1}\right) *\left(\tilde{\zeta} \mathcal{E}^{\downarrow}(\zeta) \otimes \tilde{\zeta} \mathcal{E}^{\downarrow}(\zeta)\right)\right]=\left(\bar{\lambda}_{1} * \tilde{\zeta} \mathcal{E}^{\downarrow}(\zeta)\right)\left(\tilde{\zeta} \mathcal{E}^{\downarrow}(\zeta)\right)
\end{aligned}
$$




$$
\begin{aligned}
& =\left(\bar{\lambda}_{1} * \mathcal{E}^{\downarrow}(\zeta)\right)\left(\bar{\lambda}_{1} * \tilde{\zeta}\right) \tilde{\zeta} \mathcal{E}^{\downarrow}(\zeta)=\mathcal{E}^{\uparrow}(\zeta)\left(\bar{\lambda}_{1} * \tilde{\zeta}\right)\left(\sigma_{1} * \tilde{\zeta}\right) \mathcal{E}^{\downarrow}(\zeta) \\
& =\mathcal{E}^{\uparrow}(\zeta)\left(\sigma_{1}^{\sharp} * \tilde{\zeta}\right) \mathcal{E}^{\downarrow}(\zeta),
\end{aligned}
$$

so that $\sigma_{1}^{\sharp} * \tilde{\zeta}=1$.

Lemma 4.4 For all $n \geq 1$,

$$
\sigma_{1}^{\sharp} * \zeta_{n}=2 \zeta_{n} .
$$

Proof We have

$$
\sigma_{1}^{\sharp} * \zeta_{n}=\left(\bar{\lambda}_{1} \sigma_{1}\right) * \zeta_{n}=\mu\left[\left(\bar{\lambda}_{1} \otimes \sigma_{1}\right) *\left(\zeta_{n} \otimes 1+1 \otimes \zeta_{n}\right)\right]=2 \zeta_{n} .
$$

Proposition 4.5 Let $I=\left(i_{0}, \ldots, i_{p}\right)$ be a $B$-composition of $n$, and let $\lambda=$ $\left(\lambda_{0}, \ldots, \lambda_{k}\right)$ be a B-partition of $n$.

$$
\tilde{S}^{I} * \tilde{\zeta}_{\lambda_{0}} \zeta_{\lambda_{1}} \cdots \zeta_{\lambda_{k}}= \begin{cases}0 & \text { if } \lambda \npreceq I \downarrow, \\ \left(2^{p} \prod_{j \geq 1} m_{j} !\right) \tilde{\zeta}_{i_{0}} \zeta_{i_{1}} \cdots \zeta_{i_{p}} & \text { if } \lambda=I \downarrow,\end{cases}
$$

where $m_{j}$ is the multiplicity of $j$ in $\left(i_{1}, i_{2}, \ldots, i_{p}\right)$ (not counting $\left.i_{0} !\right)$.

Proof The splitting formula yields

$$
\begin{aligned}
\tilde{S}^{I} * \tilde{\zeta}_{\lambda_{0}} \zeta_{\lambda_{1}} \cdots \zeta_{\lambda_{k}} & =\mu_{p}\left[\left(S_{i_{0}} \otimes S_{i_{1}}^{\sharp} \otimes \cdots \otimes S_{i_{p}}^{\sharp}\right) * \sum \tilde{\zeta}_{\alpha_{0}}^{\sharp} \zeta^{\alpha^{(0)}} \otimes \cdots \otimes \tilde{\zeta}_{\alpha_{p}}^{\sharp} \zeta^{\alpha^{(p)}}\right] \\
& =\sum\left(S_{i_{0}} * \tilde{\zeta}_{\alpha_{0}}^{\sharp} \zeta^{\alpha^{(0)}}\right)\left(S_{i_{1}}^{\sharp} * \tilde{\zeta}_{\alpha_{1}}^{\sharp} \zeta^{\alpha^{(1)}}\right) \cdots\left(S_{i_{p}}^{\sharp} * \tilde{\zeta}_{\alpha_{p}}^{\sharp} \zeta^{\alpha^{(p)}}\right) .
\end{aligned}
$$

By (4) and Lemma 4.3, a summand is zero if any $\alpha_{i}>0$ for $i \geq 1$, so that

$$
\tilde{S}^{I} * \tilde{\zeta}_{\lambda_{0}} \zeta_{\lambda_{1}} \cdots \zeta_{\lambda_{k}}=\sum\left(S_{i_{0}} * \tilde{\zeta}_{\alpha_{0}}^{\sharp} \zeta^{\alpha^{(0)}}\right)\left(S_{i_{1}}^{\sharp} * \zeta^{\alpha^{(1)}}\right) \cdots\left(S_{i_{r}}^{\sharp} * \zeta^{\alpha^{(p)}}\right) .
$$

If a term is nonzero in this equation, then $\lambda \preceq I \downarrow$. This proves the first case. By (4) and Lemma 4.4, $S_{i}^{\sharp} * \zeta_{i}=2 \zeta_{i}$, which proves the second case.

We are now in a position to give an explicit formula for the idempotents of [17].

Theorem 4.6 For all B-partitions $\lambda=\left(\lambda_{0}, \lambda_{1}, \ldots, \lambda_{k}\right)$ of $n$, define the elements $e_{\lambda} \in$ $\mathbf{B S y m}_{n}$ recursively by the formula

$$
e_{\lambda}=\frac{1}{2^{k} \prod_{j} m_{j} !} \tilde{S}^{\lambda} *\left(S_{n}-\sum_{\mu<\lambda} e_{\mu}\right)
$$

where $m_{j}$ is the multiplicity of $j$ in $\left(\lambda_{1}, \ldots, \lambda_{k}\right)$ (not counting $\left.\lambda_{0} !\right)$. Then

$$
e_{\lambda}=\frac{1}{\prod_{j} m_{j} !} \tilde{\zeta}_{\lambda_{0}} \zeta_{\lambda_{1}} \cdots \zeta_{\lambda_{k}}
$$


Proof Let $e_{\lambda}^{\prime}$ be the right-hand side of the above equation. By Proposition 4.5,

$$
\tilde{S}^{\lambda} * e_{\lambda}^{\prime}=\tilde{S}^{\lambda} * \frac{1}{\prod_{j} m_{j} !} \tilde{\zeta}_{\lambda_{0}} \zeta_{\lambda_{1}} \cdots \zeta_{\lambda_{k}}=\left(2^{k} \prod_{j} m_{j} !\right) e_{\lambda}^{\prime}
$$

By (47), $S_{n}=\sum e_{\lambda}^{\prime}$, where the sum ranges over all $B$-partitions $\lambda$ of $n$. Together with the above and Proposition 4.5, we have

$$
\left(2^{k} \prod_{j} m_{j} !\right) e_{\lambda}^{\prime}=\tilde{S}^{\lambda} * e_{\lambda}^{\prime}=\tilde{S}^{\lambda} *\left(S_{n}-\sum_{\mu \neq \lambda} e_{\mu}^{\prime}\right)=\tilde{S}^{\lambda} *\left(S_{n}-\sum_{\mu<\lambda} e_{\mu}^{\prime}\right)
$$

Since the $e_{\lambda}^{\prime}$ satisfy the same induction as the $e_{\lambda}$, they are equal.

Theorem 4.7 [17] The family $\left(e_{\lambda}\right)$ where $\lambda$ runs over B-partitions of $n$ forms a complete system of orthogonal idempotents for $\mathbf{B S y m}_{n}$.

\section{Idempotents in the higher order peak algebras}

Let $q$ be a primitive $r$ th root of unity. We denote by $\theta_{q}$ the endomorphism of Sym defined by

$$
\tilde{f}=\theta_{q}(f)=f((1-q) A)=f(A) * \sigma_{1}((1-q) A) .
$$

We denote by $\stackrel{\mathcal{P}}{(r)}^{(r)}$ the image of $\theta_{q}$ and by $\mathcal{P}^{(r)}$ the right $\dot{\mathcal{P}}^{(r)}$-module generated by $S_{n}$ for $n \geq 0$. Note that $\stackrel{\circ}{\mathcal{P}}^{(r)}$ is by definition a left $*$-ideal of Sym. For $r=2, \stackrel{\circ}{\mathcal{P}}^{(2)}$ is the classical peak ideal, and $\mathcal{P}^{(2)}$ is the unital peak algebra. For general $r, \stackrel{\mathcal{P}}{(r)}^{(r)}$ the higher-order peak algebra of [13], and $\mathcal{P}^{(r)}$ is its unital extension defined in [3]. These objects depend only on $r$ and not on the choice of the primitive root of unity. Bases of $\mathcal{P}^{(r)}$ can be labeled by $r$-peak compositions $I=\left(i_{0} ; i_{1}, \ldots, i_{p}\right)$ with at most one part $i_{0}$ divisible by $r$.

\subsection{The radical}

By definition, $\mathcal{P}_{n}^{(r)}$ is a $*$-subalgebra of $\mathbf{S y m}_{n}$. The radical of $\mathbf{S y m}_{n}$ consists of those elements whose commutative image is zero (see [14], Lemma 3.10). The radical of $\mathcal{P}_{n}^{(r)}$ is therefore spanned by the $S_{i_{0}} \cdot \theta_{q}\left(S^{I}-S^{I^{\prime}}\right)$ such that $I^{\prime}$ is a permutation of $I$. Indeed, the quotient of $\mathcal{P}_{n}^{(r)}$ by the span of those elements is a semisimple commutative algebra, the $*$-subalgebra of $\mathbf{S y m}_{n}$ spanned by the $p_{\lambda}(\lambda \vdash n)$ such that at most one part of $\lambda$ is multiple of $r$. This special part will be denoted by $\lambda_{0}$.

We denote by $P_{n}^{(r)}$ the subset of partitions of $n$ with at most one part divisible by $r$. The simple $\mathcal{P}_{n}^{(r)}$-modules, and the principal idempotents, can therefore be labeled by $P_{n}^{(r)}$. 


\subsection{An induction for the idempotents}

Define a total order $<$ on $P_{n}^{(r)}$ as follows: sort the partitions by decreasing length and sort partitions of the same length by reverse lexicographic order. For example,

$$
\begin{aligned}
& P_{5}^{(2)}=[11111,2111,311,41,23,5] \\
& P_{7}^{(2)}=[1111111,211111,31111,4111,2311,511,331,61,43,25,7] .
\end{aligned}
$$

Now, set

$$
e_{1^{n}}^{(r)}:=\frac{1}{n !} S_{1}^{n}
$$

and define by induction

$$
e_{\lambda}^{(r)}:=\frac{1}{m_{\lambda}} T^{\lambda} *\left(S_{n}-\sum_{\mu<\lambda} e_{\mu}^{(r)}\right)
$$

where $T_{m}=R_{m}$ if $r \mid m, T_{m}=R_{r^{i} j}$ if $r \nmid m$ and $m=i r+j$ with $0<j<r$, and $T^{\lambda}=$ $T_{\lambda_{0}} T_{\lambda_{1}} \cdots T_{\lambda_{p}}$ for $\lambda=\left(\lambda_{0} ; \lambda_{1}, \ldots, \lambda_{p}\right) \in P_{n}^{(r)}$. It follows from [13, Corollary 3.17] and from the definition of $\mathcal{P}^{(r)}$ that $T^{\lambda} \in \mathcal{P}^{(r)}$.

We want to prove that $\left(e_{\lambda}^{(r)}\right)_{\lambda \in P_{n}^{(r)}}$ is a complete system of orthogonal idempotents for $\mathcal{P}^{(r)}$.

To this aim, we introduce the sequence of (left) Zassenhaus idempotents $\zeta_{n}^{(r)}$ of level $r$ as the unique solution of the equation

$$
\sigma_{1}=\left(\sum_{p \geq 0} \zeta_{p r}^{(r)}\right) \prod_{i \geq 1, r \nmid i}^{\leftarrow} e^{\zeta_{i}^{(r)}}
$$

Note that $\zeta_{n}^{(r)}=\zeta_{n}$ for $n<2 r$.

For example, for $r=2$,

$$
\begin{aligned}
\zeta_{1}^{(2)}= & S_{1} ; \quad \zeta_{2}^{(2)}=S_{2}-\frac{1}{2} S^{11} ; \quad \zeta_{3}^{(2)}=S_{3}-S^{21}+\frac{1}{3} S^{111}, \\
\zeta_{4}^{(2)}= & S_{4}-S^{31}+\frac{1}{2} S^{211}-\frac{1}{8} S^{1111}, \\
\zeta_{5}^{(2)}= & S_{5}-S^{41}+\frac{1}{2} S^{311}-S^{23}+S^{221}-\frac{1}{2} S^{2111}+\frac{1}{2} S^{113} \\
& -\frac{1}{2} S^{1121}+\frac{1}{5} S^{11111} ;
\end{aligned}
$$

and for $r=3$,

$$
\zeta_{1}^{(3)}=S_{1} ; \quad \zeta_{2}^{(3)}=S_{2}-\frac{1}{2} S^{11} ; \quad \zeta_{3}^{(3)}=S_{3}-S^{21}+\frac{1}{3} S^{111},
$$




$$
\begin{aligned}
\zeta_{4}^{(3)}= & S_{4}-S^{31}-\frac{1}{2} S^{22}+\frac{3}{4} S^{211}+\frac{1}{4} S^{112}-\frac{1}{4} S^{1111} \\
\zeta_{5}^{(3)}= & S_{5}-S^{41}-S^{32}+S^{311}+S^{212}-\frac{2}{3} S^{2111}-\frac{1}{3} S^{1112}+\frac{1}{5} S^{11111} \\
\zeta_{6}^{(3)}= & S_{6}-S^{51}-S^{42}+S^{411}+S^{312}-\frac{2}{3} S^{3111}+\frac{1}{3} S^{222}-\frac{1}{6} S^{2211} \\
& -\frac{2}{3} S^{2112}+\frac{3}{8} S^{21111}-\frac{1}{6} S^{1122}+\frac{1}{12} S^{11211}+\frac{5}{24} S^{11112}-\frac{1}{9} S^{111111} .
\end{aligned}
$$

Define now for $\lambda=\left(\lambda_{0} ; \lambda_{1}, \ldots, \lambda_{k}\right) \in P_{n}^{(r)}$,

$$
e_{\lambda}^{\prime(r)}:=\frac{1}{m_{\lambda}} \zeta_{\lambda_{0}}^{(r)} \zeta_{\lambda_{1}}^{(r)} \cdots \zeta_{\lambda_{k}}^{(r)}
$$

We will show that $e_{\lambda}^{\prime(r)}=e_{\lambda}^{(r)}$ for all $\lambda \in P_{n}^{(r)}$. We begin with two lemmas.

\section{Lemma 5.1}

$$
\Delta\left(\zeta_{n}^{(r)}\right)= \begin{cases}1 \otimes \zeta_{n}^{(r)}+\zeta_{n}^{(r)} \otimes 1 & \text { if } r \nmid n, \\ \sum_{i=0}^{n / r} \zeta_{i r}^{(r)} \otimes \zeta_{n-i r}^{(r)} & \text { if } r \mid n .\end{cases}
$$

Proof This means that $\zeta_{n}^{(r)}$ are primitive if $r \mid n$ and that the generating series $\sum_{p \geq 0} \zeta_{r p}^{(r)}$ is grouplike. If we define new elements $Y_{p}$ by

$$
\sigma_{1}=: \prod_{p} e^{Y_{r p}} \prod_{r \nmid i}^{\leftarrow} e^{Y_{i}}
$$

the standard argument showing that the Zassenhaus elements are primitive shows as well that all the $Y_{i}$ are primitive. Now identify the first product in the right-hand side with the generating series $\sum_{p \geq 0} \zeta_{r p}^{(r)}$. Then $\zeta_{i}^{(r)}=Y_{i}$ if $r \nmid i$. Since the exponential of a primitive element is grouplike and a product of grouplike series is group-like, both products in the right-hand side above are grouplike. By identification, $\sum_{p \geq 0} \zeta_{r p}^{(r)}$ is grouplike.

Lemma 5.2 Let $\lambda=\left(\lambda_{0} ; \lambda_{1}, \ldots, \lambda_{k}\right) \in P_{n}^{(r)}$ and $I=\left(i_{0}, i_{1}, \ldots, i_{p}\right)$ be an $r$-peak composition of $n$. Then,

$$
T^{I} * \zeta_{\lambda_{0}}^{(r)} \zeta_{\lambda_{1}}^{(r)} \cdots \zeta_{\lambda_{k}}^{(r)}= \begin{cases}0 & \text { if } I \downarrow<\lambda \\ m_{I} \zeta_{i_{0}}^{(r)} \zeta_{i_{1}}^{(r)} \cdots \zeta_{i_{k}}^{(r)} & \text { if } I \downarrow=\lambda\end{cases}
$$


Proof To simplify the notation, we let $\zeta^{(r)^{\lambda}}=\zeta_{\lambda_{0}}^{(r)} \zeta_{\lambda_{1}}^{(r)} \cdots \zeta_{\lambda_{k}}^{(r)}$ for $\lambda \in P_{n}^{(r)}$. If $F^{I}=$ $F_{i_{1}} \cdots F_{i_{p}}$ with each $F_{i_{j}} \in \mathbf{S y m}_{i_{j}}$, the splitting formula and (81) yield

$$
F^{I} * \zeta^{(r)^{\lambda}}=\sum_{\substack{\lambda^{(1)} \vee \cdots \vee \lambda^{(p)}=\left(\lambda_{1}, \ldots, \lambda_{k}\right) \\ r a_{1}+\cdots+r a_{p}=\lambda_{0}}} \prod_{j=1}^{p}\left(F_{i_{j}} * \zeta_{r a_{j}}^{(r)} \zeta^{(r)^{\lambda^{(j)}}}\right)
$$

where $\lambda_{0}$ is the part (possibly 0) of $\lambda$ that is divisible by $r$, and where $\alpha \vee \beta$ denotes the partition obtained by reordering the concatenation of the partitions $\alpha$ and $\beta$.

Observe that since at most one $i_{j}$ is divisible by $r$, a product in the above summation is 0 if at least two of the partitions $\lambda^{(1)}, \ldots, \lambda^{(p)}$ are empty. If $\ell(I)>\ell(\lambda)$, then $k \leq p-2$, so this hypothesis is always satisfied. Thus,

$$
F^{I} * \zeta^{(r)^{\lambda}}=0 \quad \text { if } \ell(I)>\ell(\lambda)
$$

Suppose $I \downarrow \leq \lambda$. Then, by the definition of the order, $\ell(I) \geq \ell(\lambda)$. Hence, for $I$ such that $I \downarrow \leq \lambda$ and $\ell(I)>\ell(\lambda)$, the result follows by taking $F^{I}=T^{I}$ in (85). So suppose instead that $\ell(I)=\ell(\lambda)$. By definition, $T^{I}=T_{i_{1}} \cdots T_{i_{p}}$, where $T_{m}=R_{m}$ if $r \mid m$ and $T_{m}=R_{r^{i} j}$ if $m=i r+j$ with $0<j<r$. Since $R_{J}$ can be written as a linear combination of $S^{K}$ for which $J$ is a refinement of $K$, it follows that $T^{I}$ is equal to $S^{I}$ plus a linear combination of $S^{K}$ with $\ell(K)>\ell(I)$. By taking $F^{I}=S^{K}$ in (85), it follows that $T^{I} * \zeta^{(r)^{\lambda}}=S^{I} * \zeta^{(r)^{\lambda}}$.

It remains to show that, for $I$ and $\lambda$ of the same length, $S^{I} * \zeta^{(r)^{\lambda}}=m_{I} \zeta^{(r)^{\lambda}}$ if $I \downarrow=\lambda$ and is 0 otherwise. If $\lambda$ contains no part divisible by $r$, then it follows from (84) that if $S^{I} * \zeta^{(r)^{\lambda}} \neq 0$, we must have $I \downarrow=\lambda$, in which case $S^{I} * \zeta^{(r)^{\lambda}}=m_{I} \zeta^{(r)^{\lambda}}$.

Suppose instead that $\lambda$ contains a part that is divisible by $r$. Then each decomposition $\left(\lambda^{(1)}, \ldots, \lambda^{(p)}\right)$ in $(84)$ contains at least one $\lambda^{(j)}=\emptyset$. Thus, if $I$ contains no part that is divisible by $r$, then $S^{I} * \zeta^{(r)^{\lambda}}=0$. Otherwise, the part of $I$ that is divisible by $r$ is bounded by $\lambda_{0}$. This implies that $\lambda \leq I \downarrow$. Since we began by assuming that $I \downarrow \leq \lambda$, it follows that $I \downarrow=\lambda$, and the result follows from (84) as before.

Theorem 5.3 For all partitions $\lambda=\left(\lambda_{0} ; \lambda_{1}, \ldots, \lambda_{k}\right) \in P_{n}^{(r)}$,

$$
e_{\lambda}^{(r)}=e_{\lambda}^{\prime(r)}:=\frac{1}{m_{\lambda}} \zeta_{\lambda_{0}}^{(r)} \zeta_{\lambda_{1}}^{(r)} \cdots \zeta_{\lambda_{k}}^{(r)}
$$

Proof From the definition of $\zeta_{m}^{(r)}$ it follows that $S_{n}=\sum_{\lambda \in P_{n}^{(r)}} e_{\lambda}^{\prime(r)}$. Hence, by Lemma 5.2,

$$
m_{\lambda} e_{\lambda}^{\prime(r)}=T^{\lambda} * e_{\lambda}^{\prime}=T^{\lambda} *\left(S_{n}-\sum_{\mu \neq \lambda} e_{\mu}^{\prime(r)}\right)=T^{\lambda} *\left(S_{n}-\sum_{\mu<\lambda} e_{\mu}^{\prime(r)}\right)
$$

Thus, the elements ${e_{\lambda}^{\prime}}_{\lambda}^{(r)}$ and $e_{\lambda}^{(r)}$ satisfy the same induction equation (71). 
Theorem 5.4 The family $\left(e_{\lambda}^{(r)}\right)_{\lambda \in P_{n}^{(r)}}$ forms a complete system of orthogonal idempotents for $\mathcal{P}_{n}^{(r)}$.

Proof By construction, $e_{\lambda}^{(r)}$ are in $\mathcal{P}_{n}^{(r)}$. Their identification with $e_{\lambda}^{\prime(r)}$ shows that they are linearly independent, and (82) shows that they are orthogonal idempotents. Indeed, $Y_{i}$ are Lie idempotents of Sym, and if we write partitions $\mu$ of $n$ as $(\alpha ; \beta)$, where $\alpha_{i}$ are the parts divisible by $r$ in increasing order, and $\beta_{i}$ are the other parts in decreasing order, then

$$
Y_{\mu}=\frac{1}{m_{\alpha} m_{\beta}} Y^{\alpha} Y^{\beta}
$$

is a complete set of orthogonal idempotents of $\mathbf{S y m}_{n}$. Hence, the $e_{\lambda}^{(r)}$, which are disjoint sums of the $Y_{\mu}$, are orthogonal idempotents.

\subsection{Cartan invariants}

Recall from Sect. 5.1 that the indecomposable projective modules of $\mathcal{P}_{n}^{(r)}$ can be labeled by $\mu \in P_{n}^{(r)}$. For $\mu=\left(\mu_{0} ; \mu_{1}, \ldots, \mu_{p}\right) \in P_{n}^{(r)}$, where $\mu_{0}$ is divisible by $r$, let $\bar{\mu}=\left(\mu_{1}, \ldots, \mu_{p}\right)$ and write $\mu=\left(\mu_{0} ; \bar{\mu}\right)$.

The description of the principal orthogonal idempotents in the previous subsection shows that the dimension of the indecomposable projective module labeled by $\mu$ is equal to the number of distinct permutations of $\bar{\mu}$. We make the following conjecture:

Conjecture 5.5 The Cartan invariant $\operatorname{dim}\left(e_{v}^{(r)} * \mathcal{P}_{n}^{(r)} * e_{\mu}^{(r)}\right)$ is the number of permutations $\bar{I}$ of $\bar{\mu}$ for which the following algorithm produces $\bar{v}$.

(1) Compute the standardization $\tau=\operatorname{Std}(\bar{I})$.

(2) Replace the elements of the cycles of $\tau$ by the corresponding values in $\bar{I}$.

(3) Take the sums of the values in each cycle, discarding those that are multiples of $r$.

(4) The partition obtained by reordering these sums is $\bar{v}$.

We will prove this for the classical case $(r=2)$ in the next section. We will also prove that the matrix of the Cartan invariants of $\mathcal{P}_{n}^{(2)}$ is obtained from that of $\mathbf{B S y m}_{n}$ by merely selecting the rows and columns labeled by 2-peak partitions $P_{n}^{(2)}$. (That is, we select for the type $B$ partitions $\left(\alpha_{0} ; \alpha_{1}, \ldots, \alpha_{p}\right)$ such that $\alpha_{0}$ is even and $\alpha_{1}, \ldots, \alpha_{p}$ are odd.) We know of no such simple description, even conjectural, for the general case.

\section{The classical peak algebras $(r=2)$}

In this section, we restrict our attention to the classical peak algebra $\mathcal{P}_{n}:=\mathcal{P}_{n}^{(2)}$. We will compute the $q$-Cartan matrix of $\mathcal{P}_{n}$, thus determining the quiver of $\mathcal{P}_{n}$ and proving the conjecture at the end of the previous section for $r=2$.

Recall that for type $B$ compositions or peak compositions $I=\left(i_{0} ; i_{1}, \ldots, i_{p}\right)$, we define $\bar{I}=\left(i_{1}, \ldots, i_{p}\right)$. 


\section{1 q-Cartan invariants and quiver}

Let $\operatorname{proj}(F)=\left.F\right|_{\bar{A}=A}$ denote the projection of an arbitrary element $F \in \mathbf{M R}$ onto Sym. We will show that the projection of BSym is $\mathcal{P}$ and also that $\mathcal{P}$ can be identified with a subalgebra of BSym. This identification allows us to use known results about the quiver and $q$-Cartan invariants of BSym to study $\mathcal{P}$. Recall that the quiver can be defined as the graph whose entries of the adjacency matrix are the coefficients of $q$ in the $q$-Cartan matrix (see, e.g., [4]).

\section{Lemma 6.1}

$$
\operatorname{proj}\left(\zeta_{n}\right)=\left\{\begin{array}{ll}
\zeta_{n}^{(2)} & \text { if } n \text { is odd, } \\
0 & \text { if } n \text { is even, }
\end{array} \quad \text { and } \operatorname{proj}\left(\tilde{\zeta}_{n}\right)= \begin{cases}0 & \text { if } n \text { is odd } \\
\zeta_{n}^{(2)} & \text { if } n \text { is even }\end{cases}\right.
$$

In particular, if I is a type B composition, then

$$
\operatorname{proj}\left(e_{I}\right)= \begin{cases}e_{I}^{(2)} & \text { if I is a 2-peak composition } \\ 0 & \text { otherwise }\end{cases}
$$

Proof Recall that the elements $\zeta_{n}, \tilde{\zeta}_{n} \in \mathbf{B S y m}$ are defined as the coefficients of $t^{n}$ in the following two series, respectively,

$$
\begin{aligned}
\sigma_{t}^{\sharp} & =\left(e^{\zeta_{1} t} e^{\zeta_{2} t^{2}} e^{\zeta_{3} t^{3}} \cdots\right)\left(\cdots e^{\zeta_{3} t^{3}} e^{\zeta_{2} t^{2}} e^{\zeta_{1} t}\right), \\
\sigma_{t} & =\left(\sum_{m \geq 0} \tilde{\zeta}_{m} t^{m}\right)\left(\cdots e^{\zeta_{3} t^{3}} e^{\zeta_{2} t^{2}} e^{\zeta_{1} t}\right),
\end{aligned}
$$

where

$$
\sigma_{t}^{\sharp}:=\sum_{n \geq 0} S_{n}^{\sharp} t^{n}, \quad \sigma_{t}:=\sum_{n \geq 0} S_{n} t^{n} .
$$

The projection of $\sigma_{t}^{\sharp}$ is

$$
\mathfrak{p r o j}\left(\sigma_{t}^{\sharp}\right)=\mathfrak{p r o j}\left(\bar{\lambda}_{t} \sigma_{t}\right)=\lambda_{t} \sigma_{t},
$$

which satisfies

$$
\mathfrak{p r o j}\left(\sigma_{-t}^{\sharp}\right)=\lambda_{-t} \sigma_{-t}=\left(\lambda_{t} \sigma_{t}\right)^{-1}=\mathfrak{p r o j}\left(\sigma_{t}^{\sharp}\right)^{-1} .
$$

Combined with (90), this yields the identity

$$
\begin{aligned}
& \left(e^{-\mathfrak{p r o j}\left(\zeta_{1}\right) t} e^{\mathfrak{p r o j}\left(\zeta_{2}\right) t^{2}} e^{-\mathfrak{p r o j}\left(\zeta_{3}\right) t^{3}} \cdots\right)\left(\cdots e^{-\mathfrak{p r o j}\left(\zeta_{3}\right) t^{3}} e^{\mathfrak{p r o j}\left(\zeta_{2}\right) t^{2}} e^{-\mathfrak{p r o j}\left(\zeta_{1}\right) t}\right) \\
& \quad=\left(e^{-\mathfrak{p r o j}\left(\zeta_{1}\right) t} e^{-\mathfrak{p r o j}\left(\zeta_{2}\right) t^{2}} e^{-\mathfrak{p r o j}\left(\zeta_{3}\right) t^{3}} \cdots\right)\left(\cdots e^{-\mathfrak{p r o j}\left(\zeta_{3}\right) t^{3}} e^{-\mathfrak{p r o j}\left(\zeta_{2}\right) t^{2}} e^{-\mathfrak{p r o j}\left(\zeta_{1}\right) t}\right),
\end{aligned}
$$

from which it follows that $\mathfrak{p r o j}\left(\zeta_{2 m}\right)=0$ for all $m \geq 1$. 
From (90) and (91) we have that

$$
\begin{aligned}
\sigma_{t} & =\left(\sum_{m \geq 0} \tilde{\zeta}_{m} t^{m}\right)\left(\cdots e^{\zeta_{3} t^{3}} e^{\zeta_{2} t^{2}} e^{\zeta_{1} t}\right) \\
& =\bar{\sigma}_{-t}\left(e^{\zeta_{1} t} e^{\zeta_{2} t^{2}} e^{\zeta_{3} t^{3}} \cdots\right)\left(\cdots e^{\zeta_{3} t^{3}} e^{\zeta_{2} t^{2}} e^{\zeta_{1} t}\right),
\end{aligned}
$$

and so the generating series for the $\tilde{\zeta}_{m}$ is given by

$$
\tilde{\zeta}(t):=\sum_{m \geq 0} \tilde{\zeta}_{m} t^{m}=\bar{\sigma}_{-t}\left(e^{\zeta_{1} t} e^{\zeta_{2} t^{2}} e^{\zeta_{3} t^{3}} \cdots\right) .
$$

Denoting by $\operatorname{proj}(\tilde{\zeta})(t)$ the projection of $\tilde{\zeta}(t)$ and recalling that $\mathfrak{p r o j}\left(\zeta_{2 m}\right)=0$,

$$
\mathfrak{p r o j}(\tilde{\zeta})(-t)=\sigma_{t}\left(e^{-\mathfrak{p r o j}\left(\zeta_{1}\right) t} e^{\mathfrak{p r o j}\left(\zeta_{2}\right) t^{2}} e^{-\mathfrak{p r o j}\left(\zeta_{3}\right) t^{3}} \ldots\right)=\mathfrak{p r o j}(\tilde{\zeta})(t),
$$

where the last equality follows from (91). Thus, $\mathfrak{p r o j}(\tilde{\zeta})(t)$ is even, which implies that $\operatorname{proj}\left(\tilde{\zeta}_{2 m-1}\right)=0$ for $m \geq 1$.

Next, note that the image of the generating series in (91) is precisely the generating series of the elements $\zeta_{n}^{(r)}$ given in (72). Since this later generating series uniquely defines the elements $\zeta_{n}^{(2)}$, it follows that

$$
\operatorname{proj}\left(\tilde{\zeta}_{2 m}\right)=\zeta_{2 m}^{(2)} \quad \text { and } \operatorname{proj}\left(\zeta_{2 m+1}\right)=\zeta_{2 m+1}^{(2)}
$$

For the last assertion, recall that (64) defines $e_{I} \in \mathbf{B S y m}$ for any type $B$ composition as

$$
e_{I}=\frac{1}{m_{\bar{I}}} \tilde{\zeta}_{i_{0}} \zeta_{i_{1}} \cdots \zeta_{i_{p}}
$$

Hence, if $I$ is not a 2-peak composition (that is, if $i_{0}$ is not even or if any of $i_{1}, \ldots, i_{p}$ is not odd), then $\operatorname{proj}\left(e_{I}\right)=0$. Moreover, if $I$ is a 2-peak composition, then

$$
\mathfrak{p r o j}\left(e_{I}\right)=\frac{1}{m_{\bar{I}}} \zeta_{i_{0}}^{(r)} \zeta_{i_{1}}^{(r)} \cdots \zeta_{i_{p}}^{(r)}=e_{I}^{(2)},
$$

where the last equality is just the definition of $e_{I}^{(2)} \in \mathcal{P}$ (see (86)).

Using this result, we can prove that the peak algebra $\mathcal{P}_{n}$ is both a quotient (as proved in [1]) and a subalgebra of $\mathbf{B S y m}_{n}$.

\section{Theorem 6.2}

(i) The projection of $\mathbf{B S y m}$ onto $\mathbf{S y m}$ is $\mathfrak{p r o j}(\mathbf{B S y m})=\mathcal{P}$.

(ii) $\mathcal{P}_{n}$ is isomorphic to the subalgebra $\varepsilon_{n} * \mathbf{B S y m}_{n} * \varepsilon_{n}$ of $\mathbf{B S y m}_{n}$, where

$$
\varepsilon_{n}=\sum_{\lambda \in P_{n}^{(2)}} e_{\lambda} \in \mathbf{B S y m}_{n} .
$$


Proof By Lemma 6.1, the basis $\left(e_{I}\right)_{I}$ of BSym, where $I$ is a type $B$ composition, maps onto the basis $\left(e_{J}^{(2)}\right)_{J}$ of $\mathcal{P}$, where $J$ is a 2-peak composition. This proves (i).

Since $\operatorname{proj}\left(e_{\lambda}\right)=e_{\lambda}^{(2)}$ for 2-peak partitions $\lambda$,

$$
\operatorname{proj}\left(\varepsilon_{n}\right)=\sum_{\lambda \in P_{n}^{(2)}} e_{\lambda}^{(2)}=1 \in \mathcal{P}_{n} .
$$

Hence, $\mathfrak{p r o j}\left(\varepsilon_{n} * \mathbf{B S y m}_{n} * \varepsilon_{n}\right)=\mathcal{P}_{n}$. We now only need to prove that the two algebras have the same dimension.

For any 2-peak partition $\lambda$, the dimension of $\mathbf{B S y m}_{n} * e_{\lambda}$ is the number of rearrangements of $\bar{\lambda}$, which is also the dimension of $\mathcal{P}_{n} * e_{\lambda}^{(2)}$ (these statements follow from the facts that the elements $e_{I}$ and $e_{I}^{(2)}$, one for each 2-peak composition $I$ such that $\bar{I} \downarrow=\bar{\lambda}$, form bases of $\mathbf{B S y m}_{n} * e_{\lambda}$ and $\mathcal{P}_{n} * e_{\lambda}^{(2)}$, respectively). This implies that

$$
\begin{aligned}
\operatorname{dim}\left(\mathcal{P}_{n}\right) & =\operatorname{dim}\left(\mathcal{P}_{n} * \sum_{\lambda \in P_{n}^{(2)}} e_{\lambda}^{(2)}\right)=\operatorname{dim}\left(\mathbf{B S y m}_{n} * \varepsilon_{n}\right) \\
& \geq \operatorname{dim}\left(\varepsilon_{n} * \mathbf{B S y m}_{n} * \varepsilon_{n}\right) \geq \operatorname{dim}\left(\operatorname{proj}\left(\varepsilon_{n} * \mathbf{B S y m}_{n} * \varepsilon_{n}\right)\right)=\operatorname{dim}\left(\mathcal{P}_{n}\right),
\end{aligned}
$$

so that $\operatorname{dim}\left(\varepsilon_{n} * \mathbf{B S y m}_{n} * \varepsilon_{n}\right)=\operatorname{dim}\left(\mathcal{P}_{n}\right)$.

Corollary 6.3 Let us label the vertices of the quiver $Q_{n}$ of $\mathcal{P}_{n}$ by odd partitions $\bar{\mu}$ of $n, n-2, n-4, \ldots$. There are exactly $m>0$ arrows from $\bar{\mu}$ to $\bar{v}$ in $Q_{n}$ if and only if $\bar{v}$ is obtained from $\bar{\mu}$ by: deleting two unequal parts (in which case $m=1$ ); or merging three parts, of which at most two are equal (if no two of the merged parts are equal, then $m=2$; if exactly two are equal, then $m=1$ ).

Proof By Theorem 6.2, we can identify $\mathcal{P}_{n}$ with the subalgebra $\varepsilon_{n} * \mathbf{B S y m}_{n} * \varepsilon_{n}$ of $\mathbf{B S y m}$. Since $\varepsilon_{n}$ is a sum of primitive orthogonal idempotents of $\mathbf{B S y m}_{n}$, it follows that the quiver $Q_{n}$ of $\mathcal{P}_{n}$ is obtained from the quiver of $\mathbf{B S y m}_{n}$ by restricting to the vertices labeled by 2-peak partitions $\lambda$. The result follows immediately as the quiver of $\mathbf{B S y m}_{n}$ has already been computed [17, Theorem 9.1].

From these results we can easily derive the results mentioned at the end of the previous section for the case $r=2$.

\section{Theorem 6.4}

(i) The Cartan matrix of $\mathcal{P}_{n}$ is obtained from that of $\mathbf{B S y m}_{n}$ by selecting the rows and columns labeled by the 2-peak partitions $P_{n}^{(2)}$.

(ii) For $r=2$, Conjecture 5.5 is true. Moreover, continuing with the notation of Conjecture 5.5, I contributes $q^{\frac{1}{2}(l(\bar{\mu})-l(\bar{v}))}$ to the $q$-Cartan matrix of $\mathcal{P}_{n}$.

Proof By Lemma 6.1 and Theorem 6.2, for any $v, \mu \in P_{n}^{(2)}$, we have

$$
e_{\nu}^{(2)} * \mathcal{P}_{n} * e_{\mu}^{(2)} \cong e_{\nu} * \mathbf{B S y m}_{n} * e_{\mu}
$$


which proves (i). For the first part of (ii), we recall that a combinatorial description of the Cartan invariants of $\mathbf{B S y m}_{n}$ was given in [5]. If we restrict that description to 2-peak partitions, then the resulting formulation is equivalent to that described in Conjecture 5.5. The final assertion follows from the description of the quiver of $\mathcal{P}_{n}$ computed above.

\subsection{Comparison with earlier works}

A basis of idempotents and a complete set of orthogonal idempotents for $\mathcal{P}_{n}$ have been obtained in [2], relying on previous work on the descent algebras of type $B$ [6]. Here is a short derivation of these results, using our realization of type $B$ noncommutative symmetric functions [9].

Let us recall the notation

$$
\phi(t)=\log \sigma_{t}=\sum_{n \geq 1} \frac{\Phi_{n}}{n} t^{n} .
$$

For $r=2$,

$$
\sigma_{t}^{\sharp}:=\left.\sigma_{t}(A-q \bar{A})\right|_{q=-1}=\bar{\lambda}_{t} \sigma_{t}=\exp \left(\phi^{\sharp}(t)\right) .
$$

We have already seen that

$$
\sigma_{t}^{\sharp} * \sigma_{t}^{\sharp}=\left(\sigma_{t}^{\sharp}\right)^{2}=\exp \left(2 \phi^{\sharp}(t)\right),
$$

so that

$$
E_{\lambda}:=\sum_{I \downarrow=\lambda} \frac{\Phi^{I \sharp}}{2^{l(I)} i_{1} i_{2} \cdots i_{l(I)}}
$$

are orthogonal idempotents summing to $\sigma_{1}^{\sharp}$.

Following [9, Definition 4.14], define

$$
\eta(t):=\sigma_{t} \cdot\left[\sigma_{t}^{\sharp}\right]^{-\frac{1}{2}} .
$$

Then, $\eta(1)$ is an idempotent.

Denote by $\tilde{f}=\left.f\right|_{\bar{A}=A}$ the projection onto Sym of an element of MR. Then,

$$
\xi(t):=\tilde{\sigma}_{t}^{\sharp}=\lambda_{t} \sigma_{t}
$$

satisfies

$$
\xi(-t)=\xi(t)^{-1}
$$

Indeed,

$$
\xi(-t)=\lambda_{-t} \sigma_{-t}=\left(\lambda_{t} \sigma_{t}\right)^{-1},
$$

so that

$$
\tilde{\phi}^{\sharp}(-t)=-\tilde{\phi}^{\sharp}(t),
$$


that is, only the $\phi_{n}$ with $n$ odd survive the projection onto $\mathcal{P}$. Moreover,

$$
\begin{aligned}
\tilde{\eta}(-t) & =\sigma_{-t}\left(\lambda_{-t} \sigma_{-t}\right)^{-\frac{1}{2}}=\sigma_{-t}\left(\lambda_{t} \sigma_{t}\right)^{\frac{1}{2}} \\
& =\left[\left(\lambda_{t} \sigma_{t}\right)^{-\frac{1}{2}} \lambda_{t} \sigma_{t} \lambda_{-t}\right]^{-1}=\left[\left(\lambda_{t} \sigma_{t}\right)^{\frac{1}{2}} \lambda_{-t}\right]^{-1}=\tilde{\eta}(t),
\end{aligned}
$$

so that $\tilde{\eta}(-t)$ is even, and only the $\eta_{2 k}$ survive. Hence, if $\lambda=\left(\lambda_{0}, \lambda_{1}, \ldots, \lambda_{m}\right)=$ $\left(\lambda_{0} ; \bar{\lambda}\right)$ runs over the partitions of $n$ such that $\lambda_{0}$ is even (allowed to be 0 ) and the other parts are odd,

$$
\tilde{E}_{\lambda}=\eta_{\lambda_{0}} \sum_{J \downarrow \bar{\lambda}} \frac{\tilde{\Phi}^{I \sharp}}{2^{l(I)} i_{1} i_{2} \cdots i_{l(I)}}
$$

is a complete set of orthogonal idempotents of $\mathcal{P}_{n}$, consisting of the nonzero images of a complete set for $\mathbf{B S y m}_{n}$. These idempotents coincide with those of [2] but are different from ours. They have nevertheless a similar structure.

\section{$7 q$-Cartan invariants for generalized peak algebras}

Conjecture 5.5 provided a conjectural description of the Cartan invariants of the higher-order peak algebras $\mathcal{P}_{n}^{(r)}$. The following presents their $q$-analogs: the coefficient of $q^{k}$ in row $\lambda$, column $\mu$, is the multiplicity of the simple module $\lambda$ in the $k$ th slice of the descending Loewy series of the indecomposable projective module $\mu$. (Here $q$ is an indeterminate, not to be confused with the primitive root of unity that $q$ denoted earlier.) The usual Cartan invariants can be obtained from this $q$-analog by setting $q=1$. Note that the $q$-Cartan matrix also encodes the quiver of the algebra since the single powers of $q$ in the matrix correspond to the arrows of the quiver.

Also note that for $r \geq n$, the $q$-Cartan matrix of $\mathcal{P}_{n}^{(r)}$ is the same, up to indexation, as the $q$-Cartan matrix of $\mathbf{S y m}_{n}$. Below we write partitions in the order opposite to the order $<$ defined above, and the zero entries of the matrices are represented by dots to enhance readability.

7.1 $q$-Cartan matrices for unital peak algebras $(r=2)$

$$
\begin{aligned}
C_{2}^{(2)} & =\left(\begin{array}{ll}
1 & \cdot \\
\cdot & 1
\end{array}\right), \\
C_{3}^{(2)} & =\left(\begin{array}{lll}
1 & \cdot & \cdot \\
\cdot & 1 & . \\
\cdot & \cdot & 1
\end{array}\right), \\
C_{4}^{(2)} & =\left(\begin{array}{llll}
1 & q & \cdot & \cdot \\
\cdot & 1 & \cdot & \cdot \\
\cdot & \cdot & 1 & \cdot \\
\cdot & \cdot & \cdot & 1
\end{array}\right),
\end{aligned}
$$


$C_{5}^{(2)}=\left(\begin{array}{cccccc}1 & . & . & q & \cdot & . \\ \cdot & 1 & . & \cdot & . & . \\ \cdot & \cdot & 1 & q & . & . \\ \cdot & \cdot & . & 1 & . & . \\ \cdot & \cdot & . & . & 1 & . \\ \cdot & . & . & . & . & 1\end{array}\right)$,

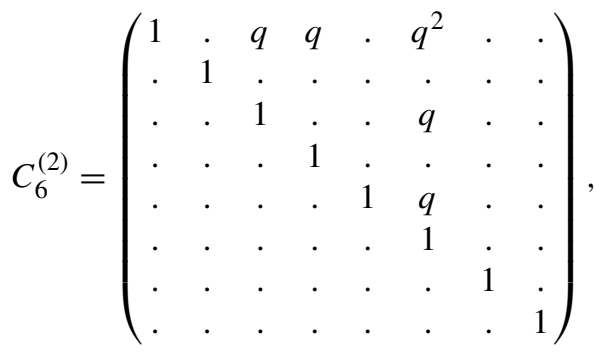

$C_{7}^{(2)}=\left(\begin{array}{ccccccccccc}1 & . & . & . & q & q & . & . & q^{2} & . & . \\ . & 1 & . & . & q & . & . & . & . & . & . \\ . & . & 1 & . & . & . & q & . & . & . & . \\ \cdot & . & . & 1 & . & q & q & . & q^{2} & . & . \\ . & . & . & . & 1 & . & . & . & . & . & . \\ . & . & . & . & . & 1 & . & . & q & . & . \\ . & . & . & . & . & . & 1 & . & . & . & . \\ . & . & . & . & . & . & . & 1 & q & . & . \\ . & . & . & . & . & . & . & . & 1 & . & . \\ . & . & . & . & . & . & . & . & . & 1 & . \\ . & . & . & . & . & . & . & . & . & . & 1\end{array}\right)$,

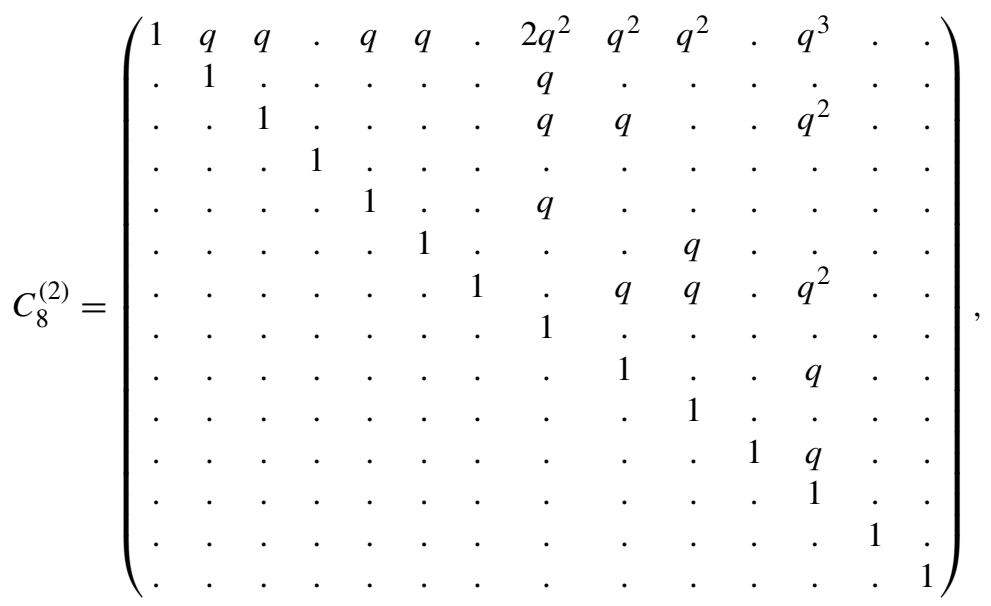




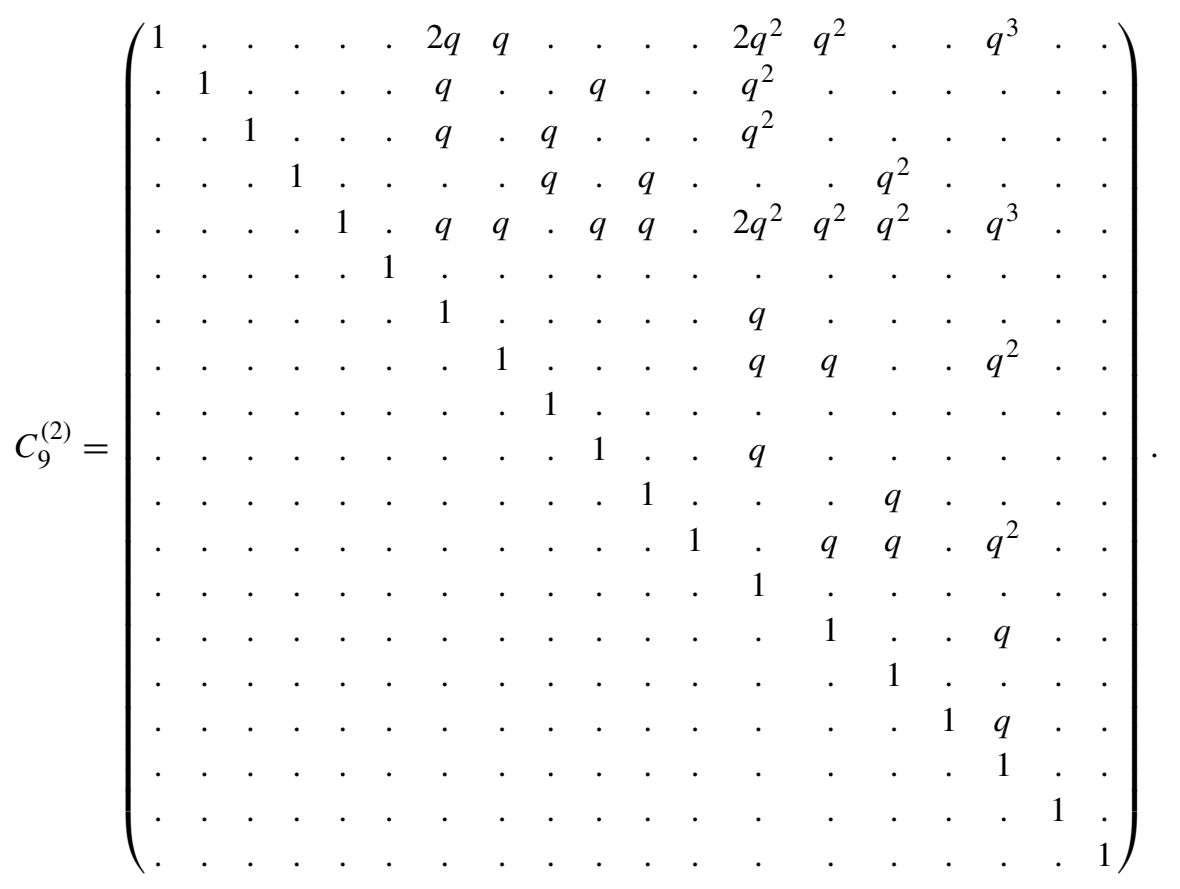

7.2 $q$-Cartan matrices for unital peak algebras of order $r=3$

$$
\begin{aligned}
& C_{3}^{(3)}=\left(\begin{array}{lll}
1 & q & \cdot \\
\cdot & 1 & \cdot \\
\cdot & \cdot & 1
\end{array}\right), \\
& C_{4}^{(3)}=\left(\begin{array}{ccccc}
1 & \cdot & \cdot & q & \cdot \\
\cdot & 1 & \cdot & \cdot & \cdot \\
\cdot & \cdot & 1 & q & \cdot \\
\cdot & \cdot & \cdot & 1 & \cdot \\
\cdot & \cdot & . & . & 1
\end{array}\right),
\end{aligned}
$$

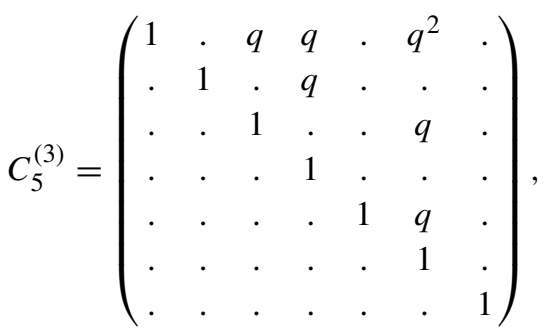


$C_{6}^{(3)}=\left(\begin{array}{cccccccccc}1 & q & q & . & q & q^{2} & 2 q^{2} & . & q^{3} & . \\ \cdot & 1 & . & . & . & . & q & . & . & . \\ \cdot & . & 1 & . & . & q & q & . & q^{2} & . \\ \cdot & . & . & 1 & . & . & . & . & . & . \\ . & . & . & . & 1 & . & q & . & . & . \\ . & . & . & . & . & 1 & . & . & q & . \\ . & . & . & . & . & . & 1 & . & . & . \\ . & . & . & . & . & . & . & 1 & q & . \\ . & . & . & . & . & . & . & . & 1 & . \\ . & . & . & . & . & . & . & . & . & 1\end{array}\right)$,

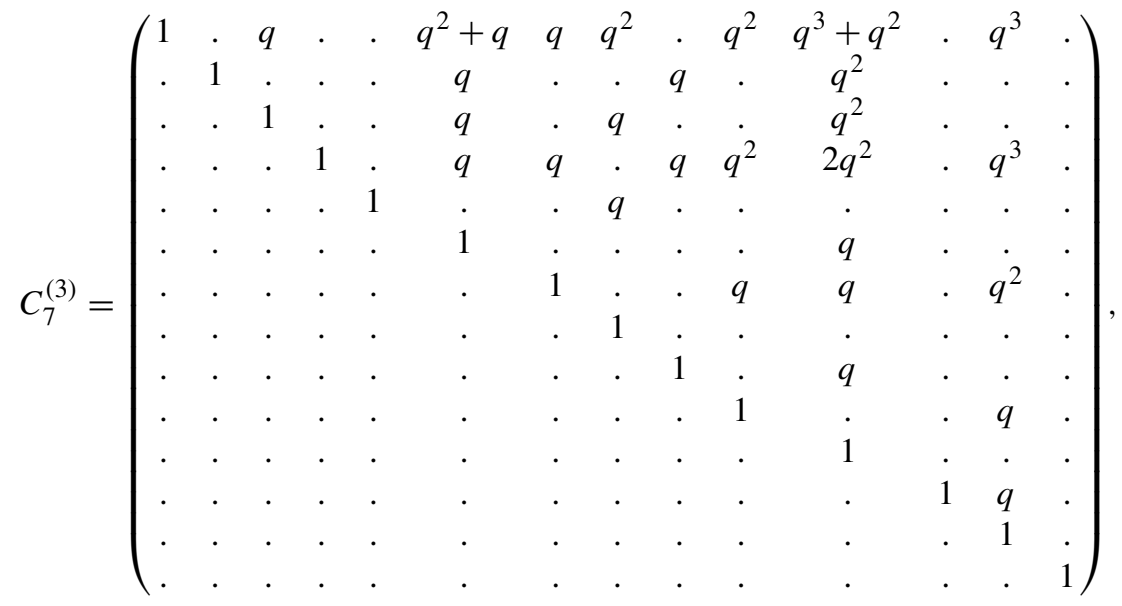

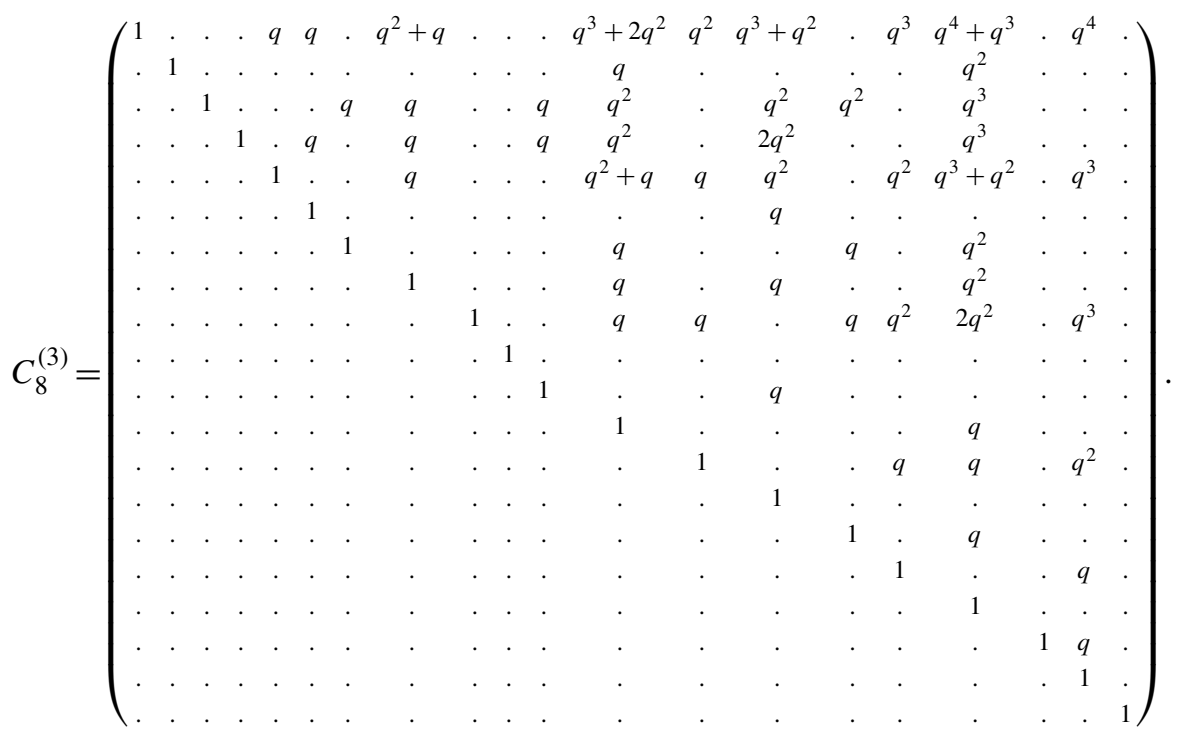


7.3 $q$-Cartan matrices for unital peak algebras of order $r=4$

$\begin{aligned} C_{4}^{(4)} & =\left(\begin{array}{ccccc}1 & \cdot & q & q^{2} & \cdot \\ \cdot & 1 & \cdot & \cdot & \cdot \\ \cdot & \cdot & 1 & q & \cdot \\ \cdot & \cdot & \cdot & 1 & . \\ \cdot & \cdot & \cdot & \cdot & 1\end{array}\right), \\ C_{5}^{(4)} & =\left(\begin{array}{ccccccc}1 & q & \cdot & q^{2} & q & q^{2} & \cdot \\ \cdot & 1 & \cdot & q & \cdot & \cdot & \cdot \\ \cdot & \cdot & 1 & \cdot & q & q^{2} & \cdot \\ \cdot & \cdot & \cdot & 1 & \cdot & \cdot & \cdot \\ \cdot & \cdot & \cdot & \cdot & 1 & q & \cdot \\ \cdot & \cdot & \cdot & \cdot & \cdot & 1 & . \\ \cdot & \cdot & \cdot & \cdot & \cdot & \cdot & 1\end{array}\right),\end{aligned}$

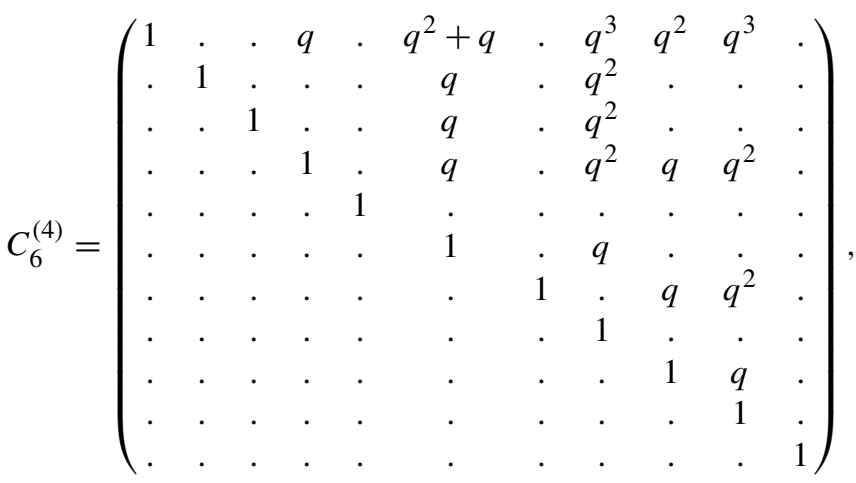

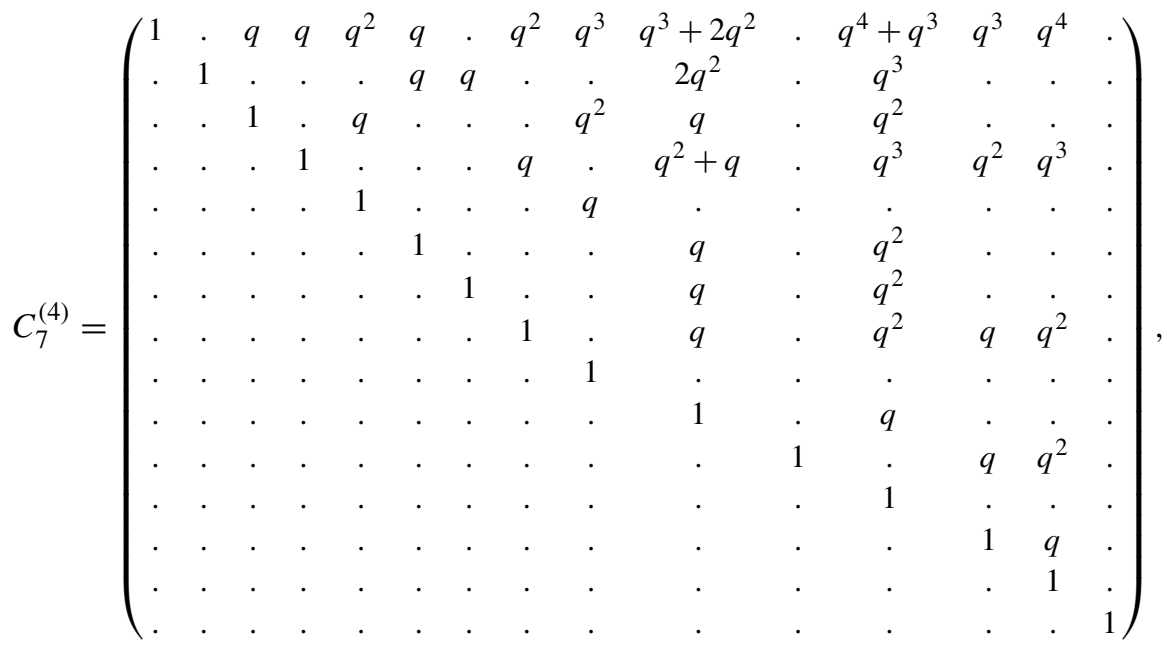




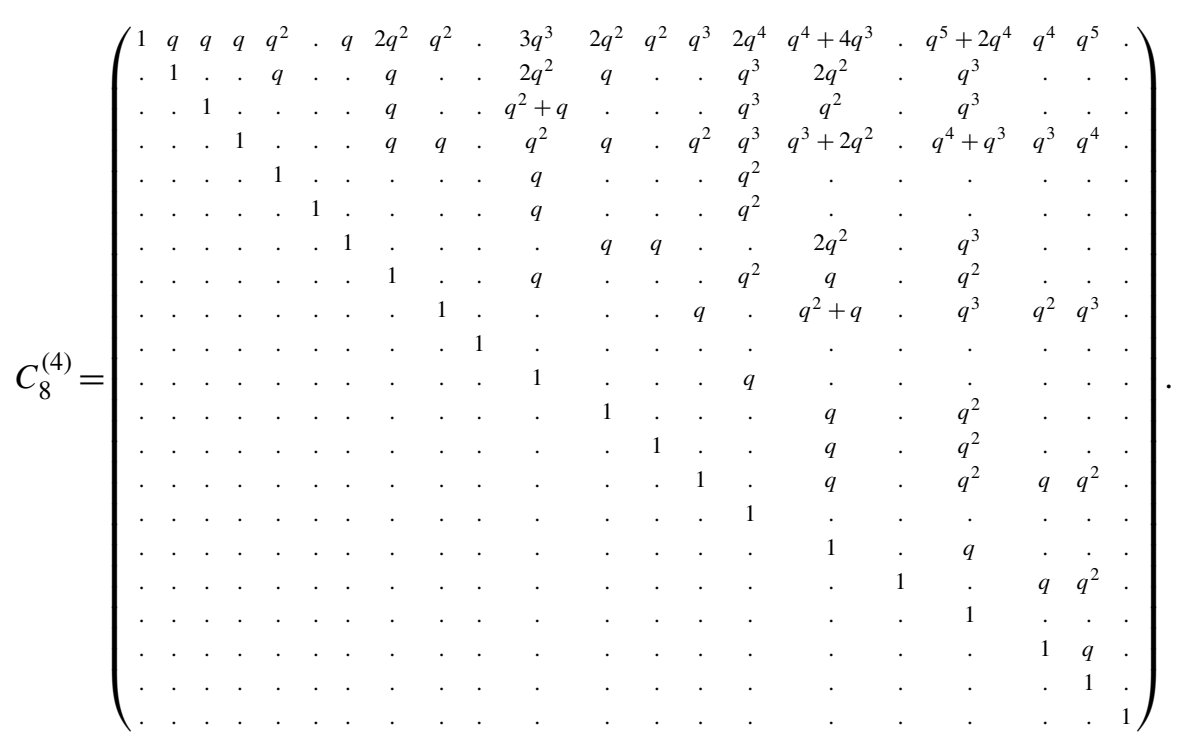

7.4 $q$-Cartan matrices for unital peak algebras of order $r=5$

$C_{5}^{(5)}=\left(\begin{array}{ccccccc}1 & q & q & q^{2} & q^{2} & q^{3} & . \\ \cdot & 1 & \cdot & q & \cdot & \cdot & \cdot \\ \cdot & \cdot & 1 & \cdot & q & q^{2} & \cdot \\ \cdot & \cdot & \cdot & 1 & \cdot & \cdot & \cdot \\ \cdot & \cdot & \cdot & \cdot & 1 & q & \cdot \\ \cdot & \cdot & \cdot & \cdot & \cdot & 1 & \cdot \\ \cdot & \cdot & \cdot & \cdot & \cdot & \cdot & 1\end{array}\right)$,

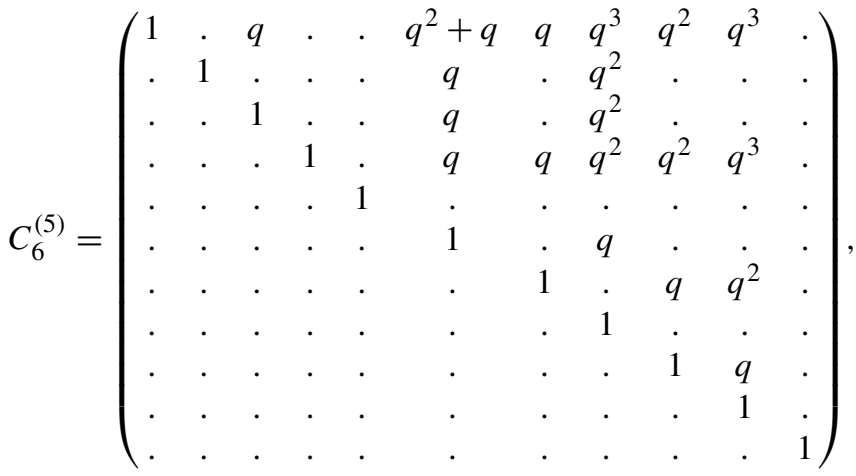




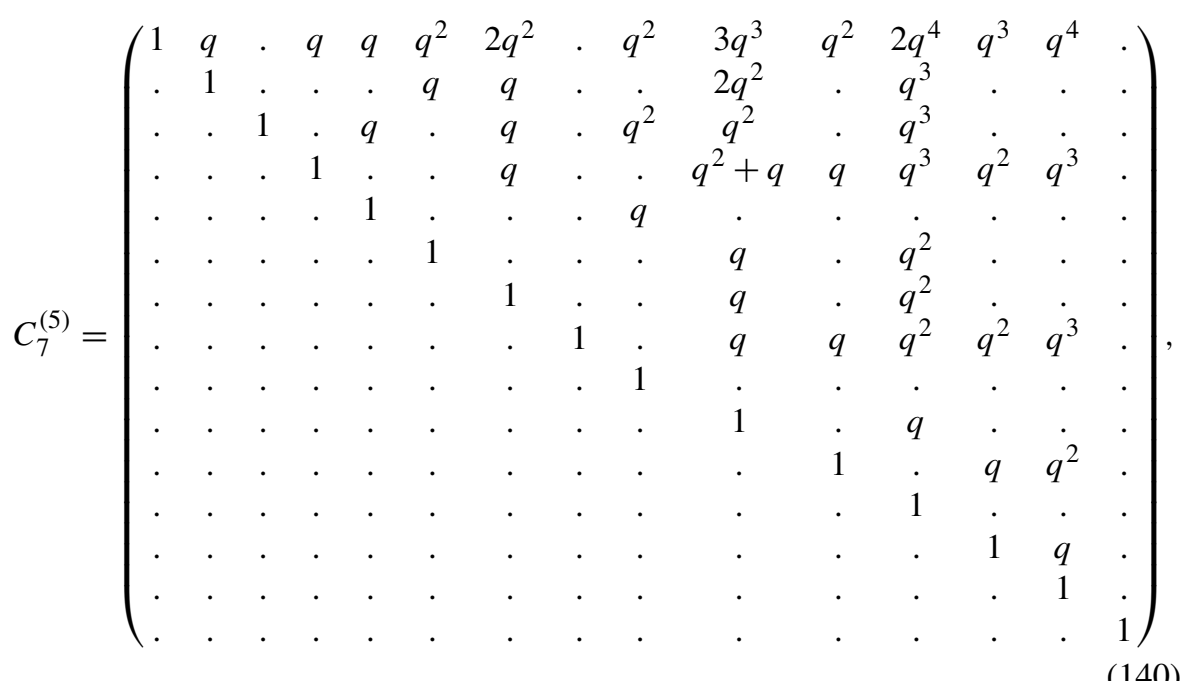

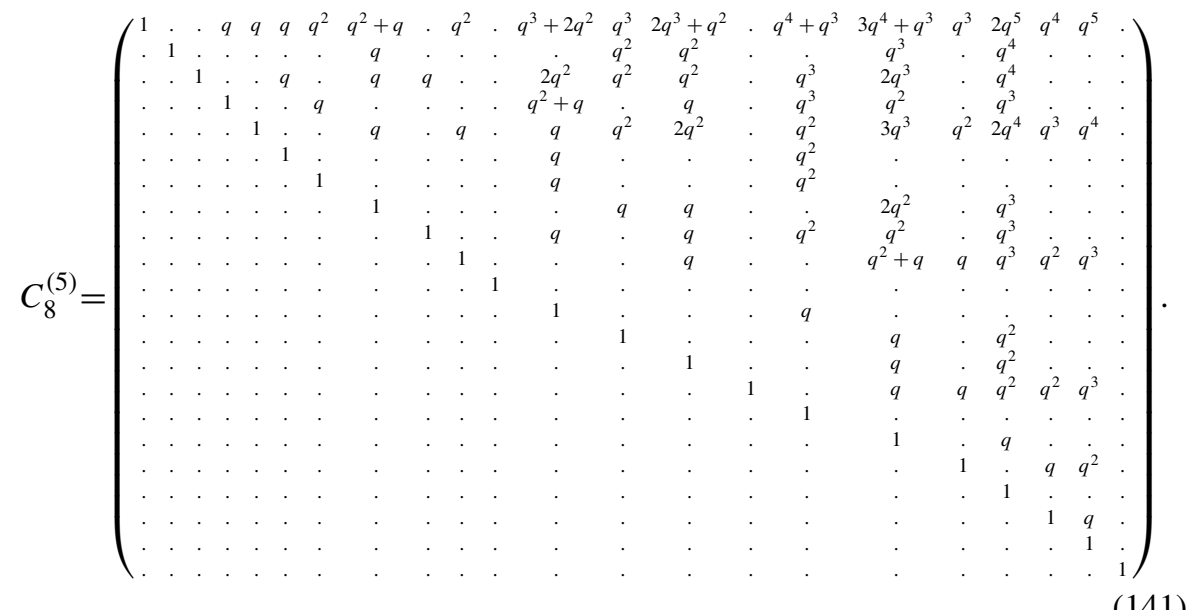

7.5 $q$-Cartan matrices for unital peak algebras of order $r=6$

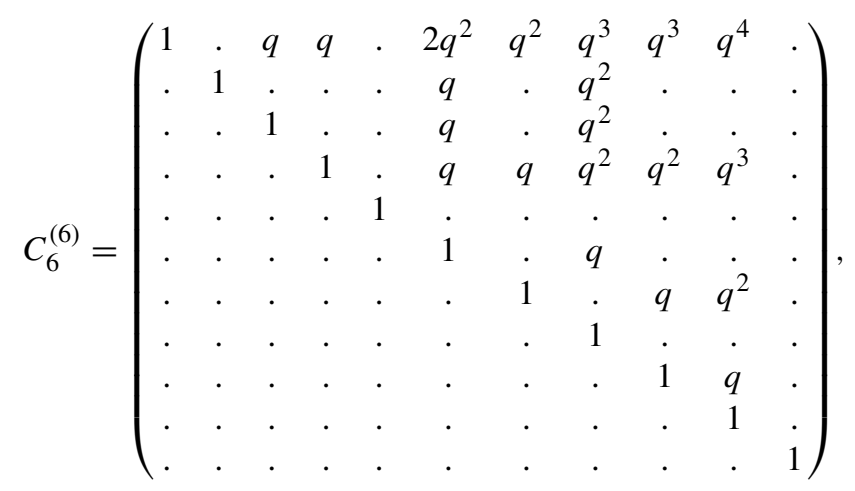




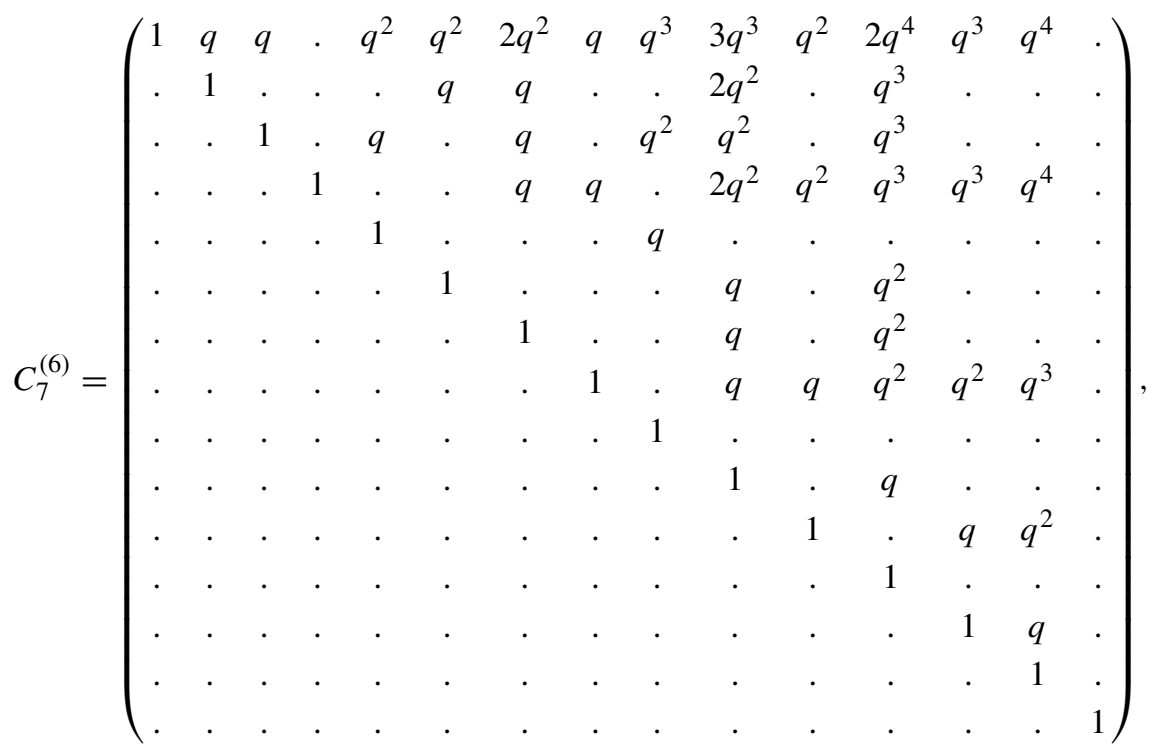

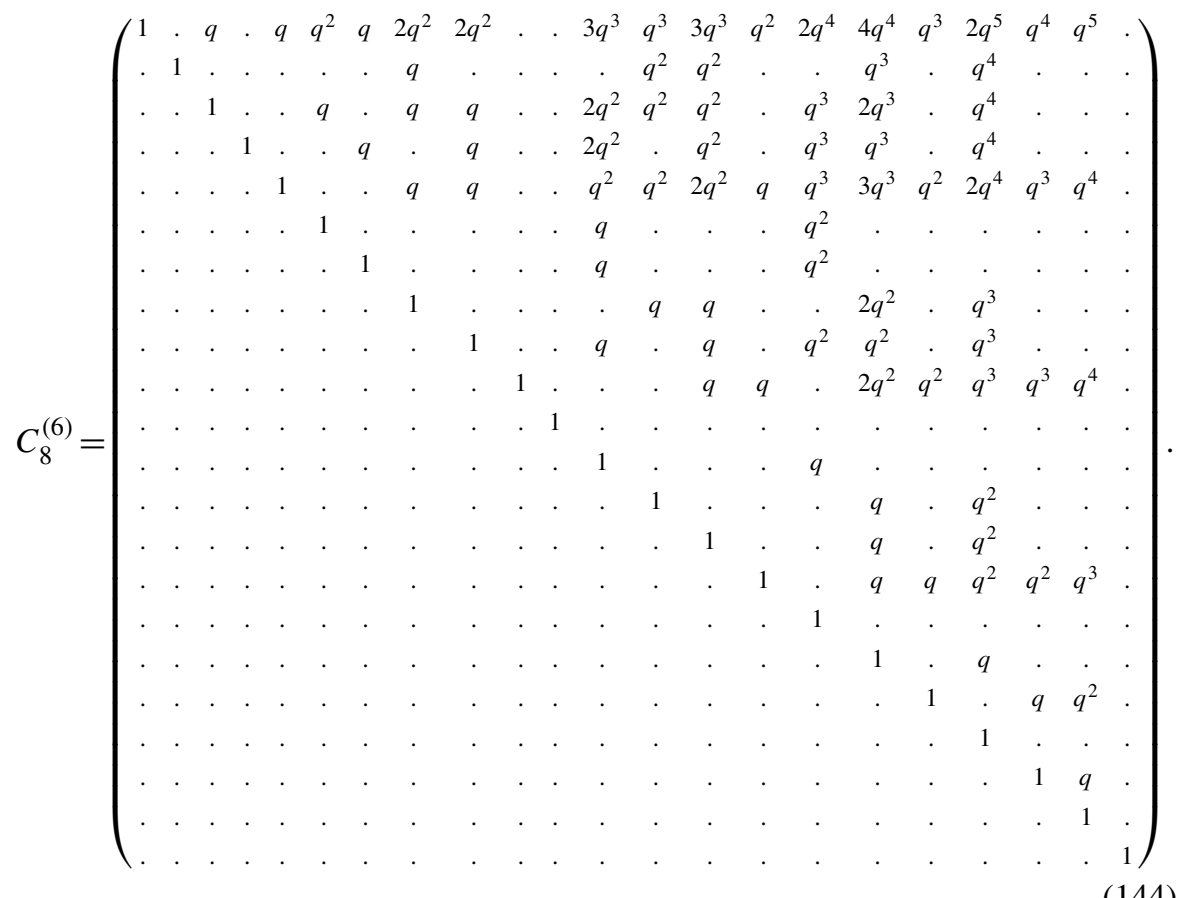


7.6 $q$-Cartan matrices for unital peak algebras of order $r=7$

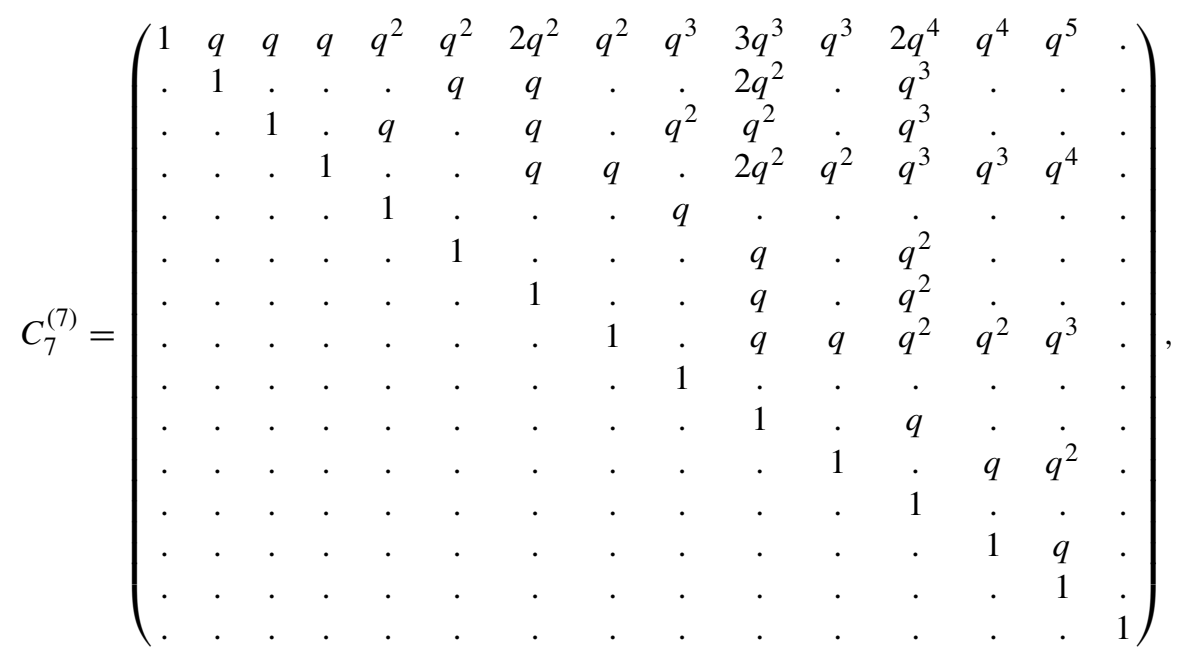

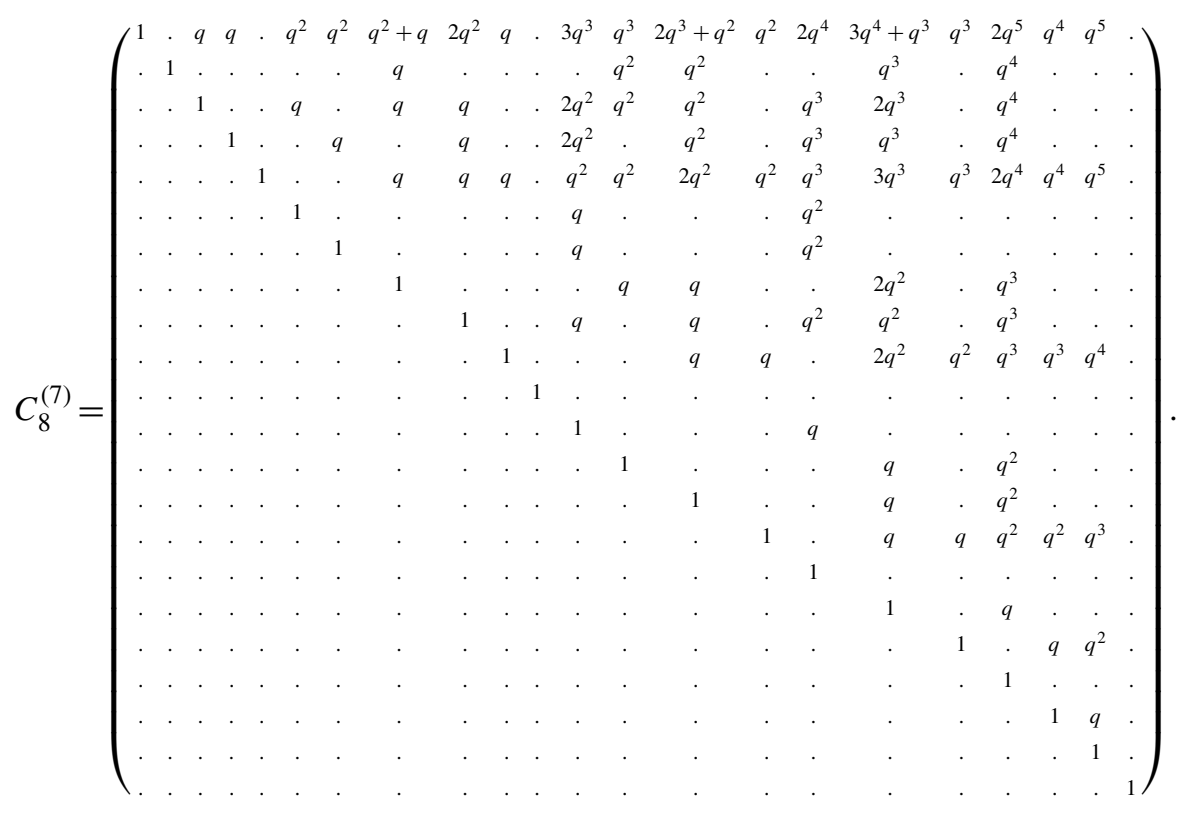

(146) 


\section{7 $q$-Cartan matrices for unital peak algebras of order $r=8$}

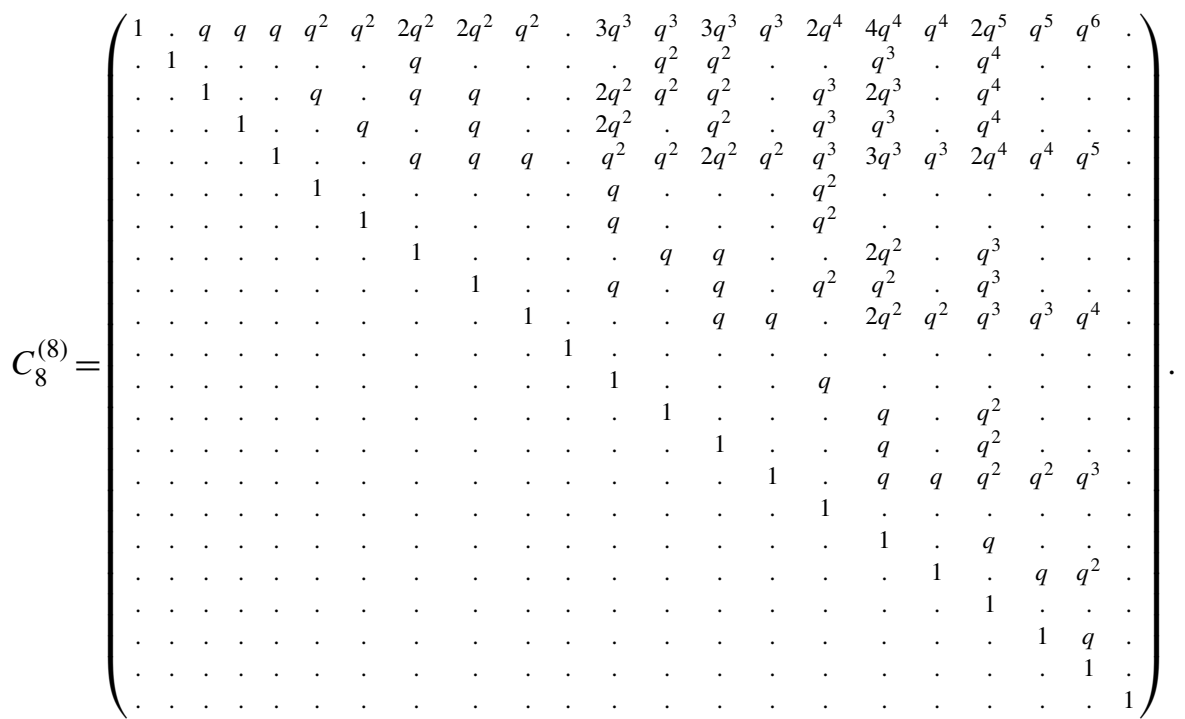

Acknowledgements This work has been partially supported by Agence Nationale de la Recherche, grant ANR-06-BLAN-0380. The authors are grateful to the contributors of both the MuPAD and Sage projects [18], and especially to those of the combinat package, for providing the development environment for this research (see [12] for an introduction to MuPAD-Combinat).

\section{References}

1. Aguiar, M., Bergeron, N., Nyman, K.: The peak algebra and the descent algebra of type B and D. Trans. Am. Math. Soc. 356, 2781-2824 (2004)

2. Aguiar, M., Nyman, K., Orellana, R.: New results on the peak algebra. J. Algebraic Comb. 23(2), 149-188 (2006)

3. Aguiar, M., Novelli, J.-C., Thibon, J.-Y.: Unital versions of the higher order peak algebras. arXiv: 0810.4634

4. Benson, D.J.: Representations and Cohomology. I: Basic Representation Theory of Finite Groups and Associative Algebras, 2nd edn. Cambridge Studies in Advanced Mathematics, vol. 30. Cambridge University Press, Cambridge (1998)

5. Bergeron, N.: A decomposition of the descent algebra of the hyperoctahedral group, II. J. Algebra 148, 98-122 (1992)

6. Bergeron, F., Bergeron, N.: Orthogonal idempotents in the descent algebra of $B_{n}$ and applications. J. Pure Appl. Algebra 79, 109-129 (1992)

7. Bergeron, N., Hivert, F., Thibon, J.-Y.: The peak algebra and the Hecke-Clifford algebras at $q=0$. J. Comb. Theory A 117, 1-19 (2004)

8. Blessenohl, D., Laue, H.: The module structure of Solomon's descent algebra. J. Aust. Math. Soc. 72(3), 317-333 (2002)

9. Chow, C.-O.: Noncommutative symmetric functions of type B. Ph.D. Thesis, MIT, 2001

10. Garsia, A.M., Reutenauer, C.: A decomposition of Solomon's descent algebra. Adv. Math. 77, 189262 (1989)

11. Gelfand, I.M., Krob, D., Lascoux, A., Leclerc, B., Retakh, V.S., Thibon, J.-Y.: Noncommutative symmetric functions. Adv. Math. 112, 218-348 (1995) 
12. Hivert, F., Thiéry, N.: MuPAD-Combinat, an open-source package for research in algebraic combinatorics. Sémin. Lothar. Comb. 51, 70 (2004) (electronic)

13. Krob, D., Thibon, J.-Y.: Higher order peak algebras. Ann. Comb. 9, 411-430 (2005)

14. Krob, D., Leclerc, B., Thibon, J.-Y.: Noncommutative symmetric functions II: Transformations of alphabets. Int. J. Algebra Comput. 7, 181-264 (1997)

15. Mantaci, R., Reutenauer, C.: A generalization of Solomon's descent algebra for hyperoctahedral groups and wreath products. Commun. Algebra 23, 27-56 (1995)

16. Novelli, J.-C., Thibon, J.-Y.: Superization and $(q, t)$-specialization in combinatorial Hopf algebras. 0803.1816 [math.CO]

17. Saliola, F.: On the quiver of the descent algebra. J. Algebra 320, 3866-3894 (2008)

18. Stein, W.A., et al.: Sage mathematics software (version 3.3). The Sage Development Team, 2009. http://www.sagemath.org 